\title{
Giant resonances observed in the scattering of 96- and 115-MeV alpha particles*
}

\author{
D. H. Youngblood, J. M. Moss, C. M. Rozsa, J. D. Bronson, A. D. Bacher, ${ }^{\dagger}$ and D. R. Brown \\ Cyclotron Institute and Physics Department, Texas A\& M University, College Station, Texas 77843
}

(Received 13 August 1975)

\begin{abstract}
Beams of 96- and $115-\mathrm{MeV} \alpha$ particles were used to study the giant resonance region in 27 nuclei ranging from ${ }^{14} \mathrm{~N}$ to ${ }^{208} \mathrm{~Pb}$. A prominent broad $(\Gamma=3-7 \mathrm{MeV})$ peak is present in the spectra at an excitation energy of $\sim 63 / A^{1 / 3} \mathrm{MeV}$ for all nuclei studied with $A \geq 36$. No such prominent peak is apparent for $A<32$. The angular distributions of the peak are characterized by an oscillatory behavior which is quite different from the monotonic behavior of the background. Distorted wave calculations with $L=2$ reproduce the experimental angular distributions quite well for most of the nuclei. Energy weighted-sum rule fractions range from 30-50\% for the lighter targets to near $100 \%$ for the heavier targets. Distorted wave calculations with a breathing mode ( $L=0$ ) form factor fail to reproduce the observed resonance strength by about a factor of 3 for all targets. The width of the peak is smallest for the closed shell nuclei ${ }^{40} \mathrm{Ca},{ }^{90} \mathrm{Zr}$, ${ }^{144} \mathrm{Sm}$, and ${ }^{208} \mathrm{~Pb}$. A small but significant broadening is observed in the rare-earth region of deformed nuclei. The giant dipole resonance is not appreciably excited for any of the targets.
\end{abstract}

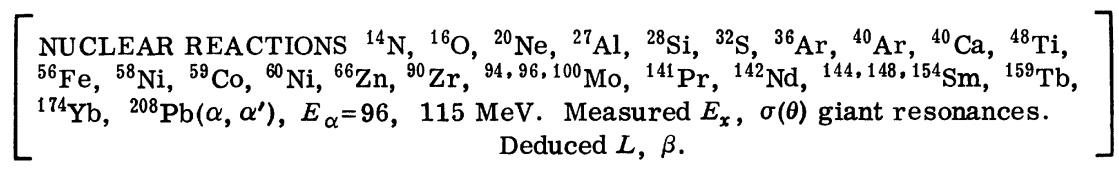

\section{INTRODUCTION}

The observation of strong, broad peaks in photonuclear excitation functions many years ago led Goldhaber and Teller $^{1}$ to propose that the nucleus was excited in a mode where essentially all of the nucleons participated in a dipole oscillation with neutron and proton spheres moving relative to one another. In the last 25 years, much study both experimentally and theoretically has been devoted to this mode of nuclear excitation, ${ }^{2}$ now characterized as the giant dipole resonance (GDR). More recently, Bohr and Mottleson ${ }^{3}$ have predicted that other modes of nuclear excitation would occur and in particular that there should be an isoscalar quadrupole giant resonance (where the protons and neutrons move together in a quadrupole shape oscillation) at an excitation energy of $\sim 58 / A^{1 / 3} \mathrm{MeV}$. Later works ${ }^{4}$ have generalized the giant resonance idea with shape, spin, and isospin modes as well as various combinations of these modes which are expected from a hydrodynamic model viewpoint. Whether the strengths of these modes are concentrated in a narrow region of excitation energy (a "giant resonance") or are spread over a wide region is an important question.

In 1971 Pitthan and Walcher ${ }^{5}$ observed in La, $\mathrm{Ce}$, and $\mathrm{Pr}$ with electron scattering experiments several peaks at high excitation in addition to the giant dipole resonance. From angular distributions one peak was tentatively identified as either $E 0$ or $E 2$ while another was tentatively assigned
$M 1$ or $M 2$. Shortly thereafter Lewis and Bertrand ${ }^{6}$ observed in inelastic proton scattering from ${ }^{27} \mathrm{~A} 1$, ${ }^{54} \mathrm{Fe},{ }^{120} \mathrm{Sn}$, and ${ }^{209} \mathrm{Bi}$ broad peaks whose excitation energies were slightly lower than that of the giant dipole resonance. They proposed that this was a combination of the GDR and a giant quadrupole resonance (GQR). Since then broad peaks have been observed just below the GDR [at $E_{x} \approx\left(60 / A^{1 / 3}\right)$ $\mathrm{MeV}]$ in many nuclei with inelastic proton, ${ }^{7}$ deuteron, ${ }^{8}{ }^{3} \mathrm{He},{ }^{9}$ and $\alpha^{10,11}$ scattering. In general, the data are fitted better if the peak is assumed to have $J^{\pi}=2^{+}$rather than $0^{+}$, and somewhat more than half of the E2 energy weighted sum rule is exhausted by the peak. Attempts ${ }^{12}$ to observe this strength with either $(p, \gamma)$ or $(\alpha, \gamma)$ reactions (in $s d$ shell and lighter nuclei) were not successful. However, these reactions are sensitive only to strength in the $p_{0}$ (or $\alpha_{0}$ ) channels. Recently Meyer-Schützmeister et al. ${ }^{13}$ have observed with the ${ }^{54} \mathrm{Fe}(\alpha, \gamma){ }^{58} \mathrm{Ni}$ reaction a peaking of $E 2$ strength consistent with the inelastic scattering results.

In order to resolve many apparent conflicts among existing data and to establish systematic features of this resonance we have studied the giant resonance region for many nuclei from ${ }^{14} \mathrm{~N}$ to ${ }^{208} \mathrm{~Pb}$ by inelastic $\alpha$ scattering. The $\alpha$ particle has $S=0$ and $T=0$ so that only isoscalar natural parity transitions are strongly excited. In numerous studies of low-lying collective states $\alpha$ inelastic scattering has proven to be a valuable spectroscopic tool. ${ }^{14}$ Additionally, $\alpha$ angular distributions are characterized by diffraction structure which 
is indicative of the angular momentum transferred to the nucleus. Such structure should aid in separation of the peak from the nuclear continuum and in determining which multipolarities are present in the peak at $60 / A^{1 / 3} \mathrm{MeV}$. Some of the results of these investigations have been reported earlier. ${ }^{11,15}$

\section{EXPERIMENTAL PROCEDURE}

Beams of $96-$ and $115-\mathrm{MeV} \alpha$ particles from the Texas A \& $\mathrm{M}$ cyclotron were used to bombard either gas or solid targets. The total charge was collected and integrated in a shielded Faraday cup located about $3 \mathrm{~m}$ behind the target position. Two $\triangle E-E$ silicon detector telescopes with a constant angular separation of $5^{\circ}$ for solid targets $\left(10^{\circ}\right.$ for gas targets) were used to detect the inelastically scattered $\alpha$ particles. Surface barrier detectors $0.7-\mathrm{mm}, 1-\mathrm{mm}$, and $2-\mathrm{mm}$ thick $(\Delta E)$ and $\mathrm{Si}(\mathrm{Li})$ detectors 3-mm $(E)$ thick were used. For runs at $115 \mathrm{MeV}$ a $2-\mathrm{mm} \Delta E$ was always used, as $\sim 4.6 \mathrm{~mm}$ of $\mathrm{Si}$ is required to stop the elastically scattered particles. The over-all energy resolution varied from 100 to $400 \mathrm{keV}$ full width at half maximum (FWHM) and was determined primarily by kinematic spread of the scattered $\alpha$ particles over the $\sim 1^{\circ}$ angular resolution of the detection system. After amplification, pulses from the detectors were routed through linear gates into electronic particle identifiers. The linear gates were opened only when pulses occurred in both the $\Delta E$ and $E$ detectors. Single channel analyzers were used to select pulses corresponding to $\alpha$ particles and supplied a signal to gate the $\Delta E+E$ signal into a biased amplifier and then to analog-to-digital convertors interfaced to a PDP 15-40 computer. Two-dimensional spectra of particle identifier output vs total energy were checked to insure that the particle identifier output was independent of energy. Energy calibrations spanning the giant resonance region were obtained from inelastic $\alpha$ scattering on ${ }^{12} \mathrm{C}$ at angles from $10^{\circ}$ to $60^{\circ}$.

In order to reduce tailing of the elastic peak, the solid angle defining collimator was placed between the $\Delta E$ and $E$ detectors and the collimators were polished to ensure squareness and uniformity. The detectors were shielded so that they could see neither the entrance nor exit ports of the chamber at the most extreme angles run. The gas cell collimator did not permit observation of either entrance or exit windows of the cell. The beam passed through an $x-y$ collimator at the exit of the cyclotron, then through the $165^{\circ}$ analyzing magnet and another $x-y$ collimator about $2 \mathrm{~m}$ before a $30^{\circ}$ switching magnet. The first $x-y$ collimator determined the initial object size (and hence the image size on target, typically $1.5 \mathrm{~mm}$ wide by $3 \mathrm{~mm}$ high), whereas the last $x-y$ collimator caught extraneous beam which, although very low in intensity, could cause significant background problems in the region of interest. There were no collimators after the switching magnet. The beam was initially tuned visually to produce the desired spot size. Then with an empty target holder in place and a detector at $15^{\circ}$, very fine tuning and adjustments of the $x-y$ slits were made to minimize the backg round count rate.

Solid targets were self-supporting foils ranging in thickness from 0.4 to $20 \mathrm{mg} / \mathrm{cm}^{2}$, generally enriched to greater than $95 \%$ in the desired isotope. The target thickness was usually determined by weighing and checked by measuring the energy loss of $\alpha$ particles from an ${ }^{241} \mathrm{Am}$ source. For targets with thickness greater than several $\mathrm{mg} / \mathrm{cm}^{2}$ (all targets with $A \approx 141$ and ${ }^{56} \mathrm{Fe}$ ), the energy loss technique could not be used. For ${ }^{40} \mathrm{Ca}$ weighing was impractical due to the oxidation rate. Both $1.5-\mathrm{cm}$ round and $2.2-\mathrm{cm}$ square target foils were used depending on the type of target. The target holder was shielded with $0.1-\mathrm{cm}$ thick Ta which ensured that no $\alpha$ particles which hit the target frame had sufficient energy after scattering to reach the $E$ detector. Gaseous targets $\left(\mathrm{N}_{2}, \mathrm{O}_{2}, \mathrm{Ne}, \mathrm{SiH}_{4}, \mathrm{H}_{2} \mathrm{~S}, \mathrm{Ar}\right)$ were confined to a cylindrical cell $8.9 \mathrm{~cm}$ in diameter and 2.5 $\mathrm{cm}$ high covered with $2.5-\mu \mathrm{m}$ thick Havar foil. The pressure was monitored with a mercury manometer and remained essentially constant for all runs.

Several checks were performed to ascertain the contribution of any nontarget background. With the ion source arc off, the detectors were positioned at $0^{\circ}$ in the remaining very low-intensity beam, and spectra accumulated (Fig. 1). In this manner contributions from both beam tailing and reactions in the detector were measured simultaneously. In the region of the spectra corresponding to $E_{\text {exc }} \gtrless 9 \mathrm{MeV}$, less than 10 counts/ channel were present, compared with several thousand for an equivalent "real" spectrum. Spectra were accumulated at several angles with an empty target frame to ascertain contributions due to frame scattering. The yield over the entire range was negligible compared to that with a target in place. Spectra were accumulated with an empty gas cell (negligible contribution over the angular range studied) and with a ${ }^{4} \mathrm{He}$ gas target (Fig. 1). ${ }^{4} \mathrm{He}$ has no excited states below $20 \mathrm{MeV}$ and thus serves as an excellent test of the overall cleanliness of the beam, gas cell, and detector collimator system. In each case there was negligible yield in the region corresponding to $E_{\text {exc }} \approx 8-18 \mathrm{MeV}$. All of the beam quality checks 
were done for each accelerator run during which data were taken. During several runs the checks were performed at the beginning, middle, and end of the run.

\section{EXPERIMENTAL RESULTS AND DATA ANALYSIS}

The reactions studied and the number of spectra taken for each are listed in Table I. Spectra from all targets studied are shown in Fig. 2. For $A \geqslant 36$, the spectra are characterized by a welldefined broad peak (which we shall call the GQR) at an excitation energy of about $63 / A^{1 / 3} \mathrm{MeV}$ (Fig. 3) sitting on a large $\alpha$-particle continuum. This peak is not apparent ${ }^{15}$ for targets with $A<32$. In several of the spectra taken at $\theta_{\mathrm{lab}}<15^{\circ} \mathrm{a}$ broad peak from $H(\alpha, \alpha) H$ is apparent. Peaks due to inelastic scattering from carbon and oxygen con-
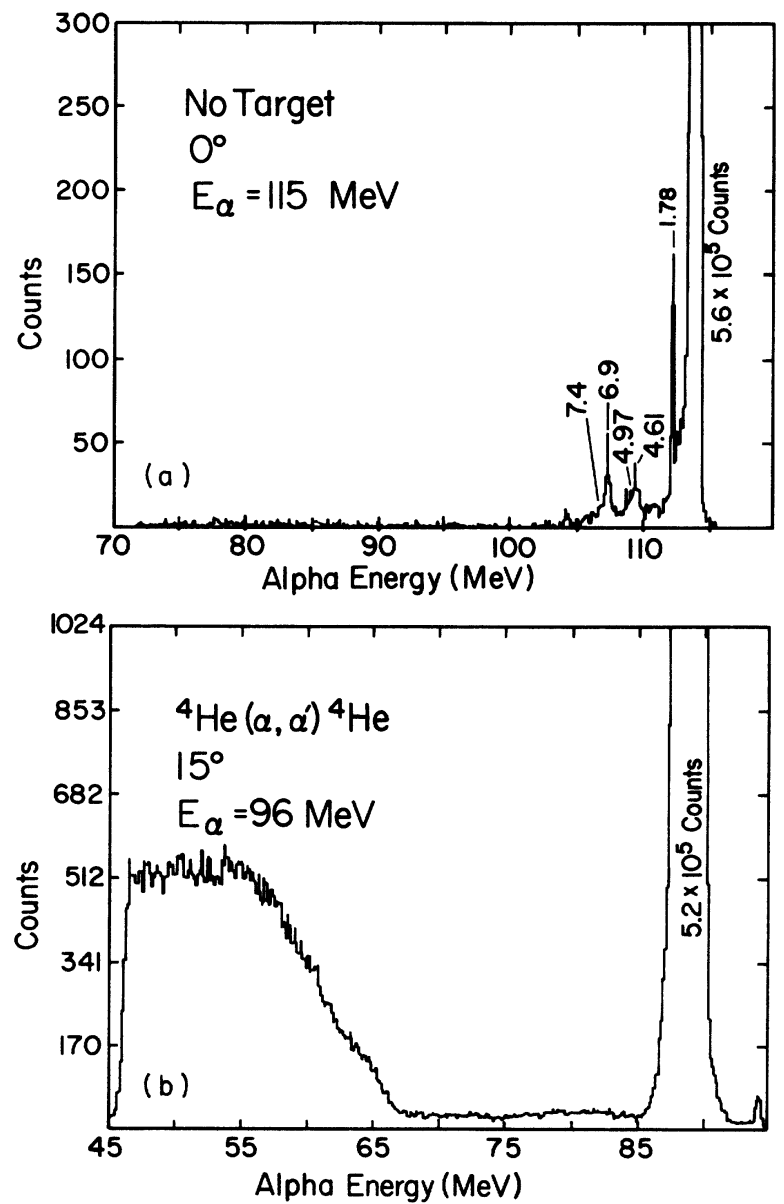

FIG. 1. (a) $\alpha$ spectrum taken at $0^{\circ}$ with the ion-source arc current off. There are $5.6 \times 10^{5}$ counts in the "elastic" peak. Peaks due to ${ }^{28} \mathrm{Si}\left(\alpha, \alpha^{\prime}\right)$ reactions in the detector are indicated. (b) ${ }^{4} \mathrm{He}\left(\alpha, \alpha^{\prime}\right){ }^{4} \mathrm{He}$ spectrum taken with ${ }^{4} \mathrm{He}$ in the gas cell. taminants on the target are present in the spectra for several nuclei and are labeled. Structure apparent on the low energy side of the GQR peak in ${ }^{36} \mathrm{Ar},{ }^{56} \mathrm{Fe},{ }^{58} \mathrm{Ni}$, and ${ }^{59} \mathrm{Co}$ tracks kinematically with the GQR. The GQR peak is more prominent relative to the continuum in spectra taken at $E_{\alpha}$ $=115 \mathrm{MeV}$ than in those taken at $96 \mathrm{MeV}$. The continuum may be due in part to excitation of other states in the target nucleus and preequilibrium processes in which an $\alpha$ particle is emitted. Since the GQR is generally above $n, p$, and $\alpha$ particle decay thresholds, processes involving three-body final states such as nucleon and particle knockout, and pickup reactions involving unstable particles $\left({ }^{5} \mathrm{Li}\right.$ or $\left.{ }^{5} \mathrm{He}\right)$ are likely to contribute significantly to the continuum. The latter process has been studied $^{16}$ for heavier nuclei at somewhat lower bombarding energies and found to contribute a significant portion of the yield in the region above the GQR. In Fig. 4 spectra for ${ }^{40} \mathrm{Ca},{ }^{94} \mathrm{Mo}$, and ${ }^{208} \mathrm{~Pb}$ taken over a wide excitation range indicate the strong contribution of the pickup process for ${ }^{208} \mathrm{~Pb}$ and the declining importance of it for the lighter nuclei. In most of the spectra shown in Fig. 2 for $A>40$, there is a weak broad bump ly-

TABLE I. Targets studied with inelastic $\alpha$ scattering.

\begin{tabular}{|c|c|c|c|}
\hline Target & Form & $\begin{array}{c}E_{\alpha} \\
(\mathrm{MeV})\end{array}$ & $\begin{array}{l}\text { No. of } \\
\text { spectra } \\
\text { taken }\end{array}$ \\
\hline${ }^{14} \mathrm{~N}$ & $\mathrm{~N}_{2}$ & 96 & 6 \\
\hline${ }^{16} \mathrm{O}$ & $\mathrm{O}_{2}$ & 96 & 6 \\
\hline${ }^{20} \mathrm{Ne}$ & $\mathrm{Ne}$ & 96 & 6 \\
\hline${ }^{27} \mathrm{Al}$ & foil & 96 & 8 \\
\hline${ }^{28} \mathrm{Si}$ & $\mathrm{SiH}_{4}$ & 96 & 6 \\
\hline${ }^{32} \mathrm{~S}$ & $\mathrm{H}_{2} \mathrm{~S}$ & 96 & 19 \\
\hline${ }^{36} \mathrm{Ar}$ & $\mathrm{Ar}$ & 96 & 12 \\
\hline${ }^{40} \mathrm{Ar}$ & $\mathrm{Ar}$ & 96 & 12 \\
\hline${ }^{40} \mathrm{Ca}$ & foil & 96,115 & 18,8 \\
\hline${ }^{48} \mathrm{Ti}$ & foil & 96 & 3 \\
\hline${ }^{56} \mathrm{Fe}$ & foil & 96 & 15 \\
\hline${ }^{58} \mathrm{Ni}$ & foil & 115 & 10 \\
\hline${ }^{59} \mathrm{Co}$ & foil & 115 & 2 \\
\hline${ }^{60} \mathrm{Ni}$ & foil & 96,115 & 4,10 \\
\hline${ }^{66} \mathrm{Zn}$ & foil & 115 & 2 \\
\hline${ }^{90} \mathrm{Zr}$ & foil & 96 & 15 \\
\hline${ }^{94} \mathrm{Mo}$ & foil & 115 & 4 \\
\hline${ }^{96} \mathrm{Mo}$ & foil & 115 & 2 \\
\hline${ }^{100} \mathrm{Mo}$ & foil & 96,115 & 4,2 \\
\hline${ }^{141} \mathrm{Pr}$ & foil & 115 & 3 \\
\hline${ }^{142} \mathrm{Nd}$ & foil & 115 & 10 \\
\hline${ }^{144} \mathrm{Sm}$ & foil & 115 & 8 \\
\hline${ }^{148} \mathrm{Sm}$ & foil & 115 & 13 \\
\hline${ }^{154} \mathrm{Sm}$ & foil & 115 & 14 \\
\hline${ }^{159} \mathrm{~Tb}$ & foil & 115 & 2 \\
\hline${ }^{174} \mathrm{Yb}$ & foil & 96,115 & 12,4 \\
\hline${ }^{208} \mathrm{~Pb}$ & foil & 96,115 & 17,18 \\
\hline
\end{tabular}



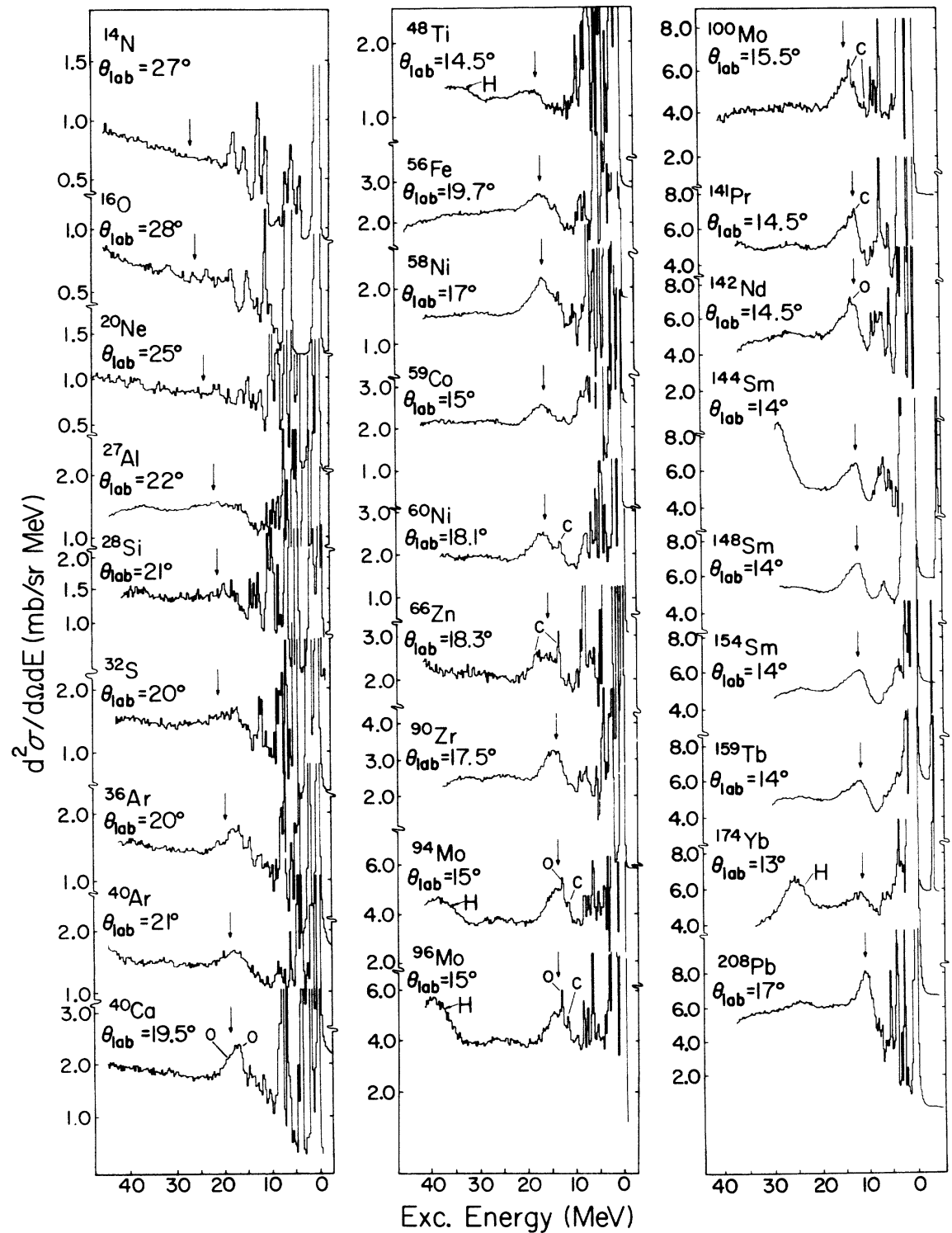

FIG. 2. Inelastic $\alpha$ spectra from each of the nuclei studied. The arrow marks $E_{x} \approx\left(63 / A^{1 / 3}\right) \mathrm{MeV}$. In several spectra the $\mathrm{H}\left(\alpha, \alpha^{\prime}\right) \mathrm{H}$ peak may be seen to the left of the GQR. Contaminant peaks in the vicinity of the GQR are indicated. The bombarding energy was $96 \mathrm{MeV}$ for all targets with $A \leq 56$ and for ${ }^{90} \mathrm{Zr}$. All others were taken at $E_{\alpha}=115 \mathrm{MeV}$.

ing at 20-30 MeV excitation, which is most probably from this process.

Since present theories are unable to account quantitatively for the continuum, it was treated as background and an empirical subtraction procedure was used. First an angle was chosen where the GQR was near a minimum. A power series fit was then made to a curve which smoothly joined the backg round above the GQR (flat) to that below (rising). The resulting function was renormalized above the GQR for other angles and subtracted from the data. This procedure is illustrated in Fig. 5. The reliability of this method

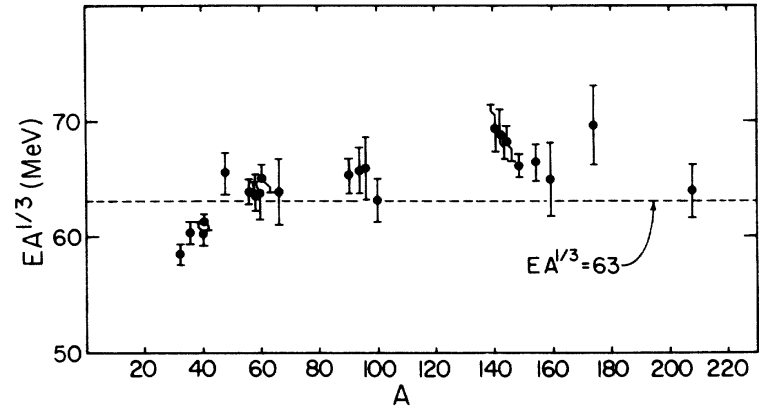

FIG. 3. The excitation energy of the GQR multiplied by $A^{1 / 3}$ is plotted vs $A$. 
depends on two criteria: (1) the contrast between the monotonic angular distribution of the continuum [Fig. 6(a)] and the diffraction structure observed for the GQR; (2) the observation of a fairly symmetric peak sitting on a background which lends itself to interpolation. Criterion (2) is difficult to specify quantitatively. It is apparent

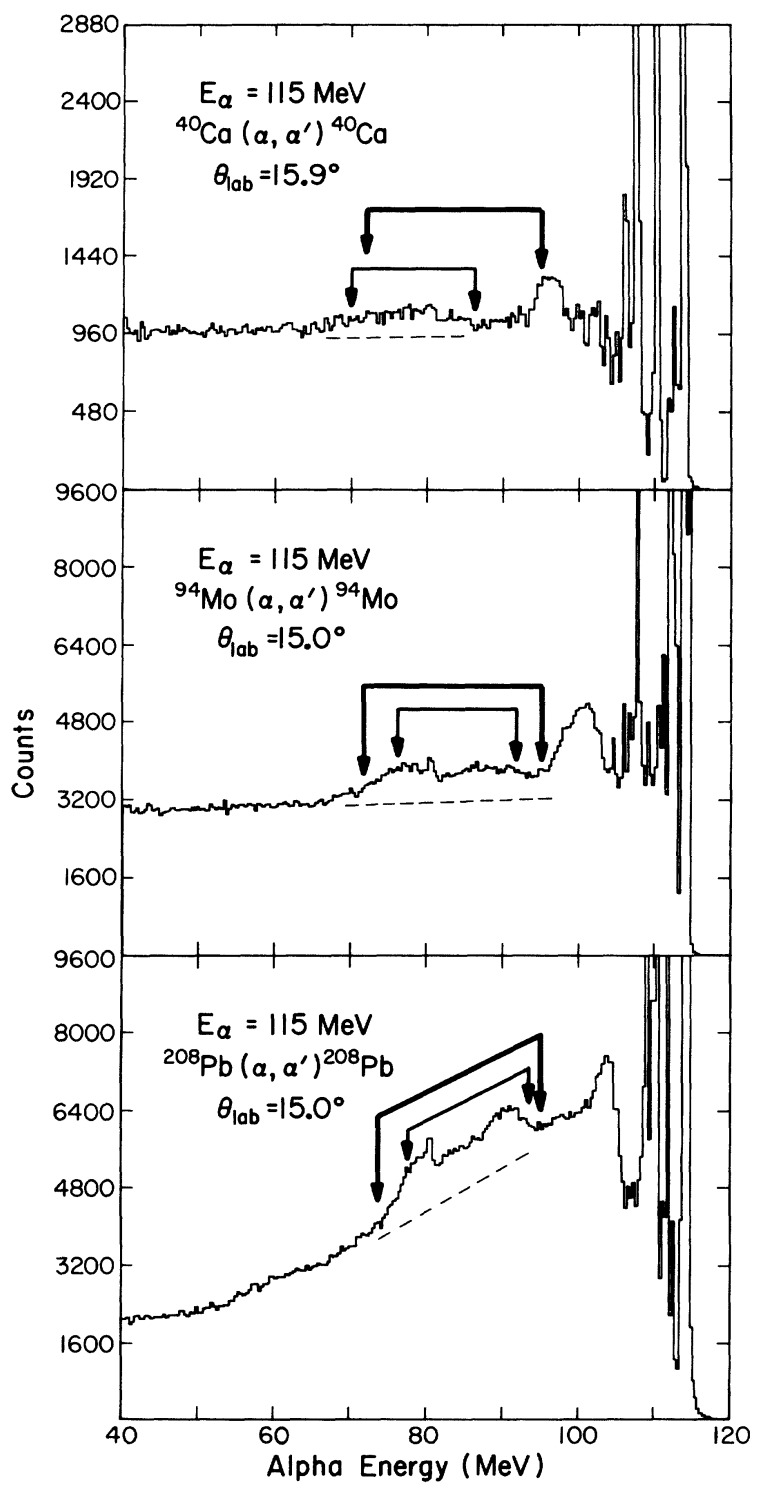

FIG. 4. Spectra from ${ }^{40} \mathrm{Ca},{ }^{94} \mathrm{Mo}$, and ${ }^{208} \mathrm{~Pb}$ taken over a wide excitation range to show the background trends. The kinematic limits for ${ }^{5} \mathrm{Li}$ and ${ }^{5} \mathrm{He}$ breakup contributions are indicated by broad and narrow lines, respectively. The discontinuity at $E_{\alpha} \approx 82 \mathrm{MeV}$ is due to a detector dead layer, as two detectors were summed to obtain the $E$ signal for these spectra. The dashed lines suggest the background trends in the absence of the ${ }^{5} \mathrm{He}$ and ${ }^{5} \mathrm{Li}$ plateau. however in nuclei with $A<36$ that the presence of fine structure and the broadening of the GQR peak make the separation of peak from background considerably more ambiguous than in medium and heavy mass nuclei. Figure $6(\mathrm{~b})$ shows a case where the above criteria are satisfied, and another case where they are not.

Target contaminant contributions (primarily ${ }^{12} \mathrm{C}$ and ${ }^{16} \mathrm{O}$ ) appear as narrow peaks superimposed on the broad GQR structure and were subtracted from

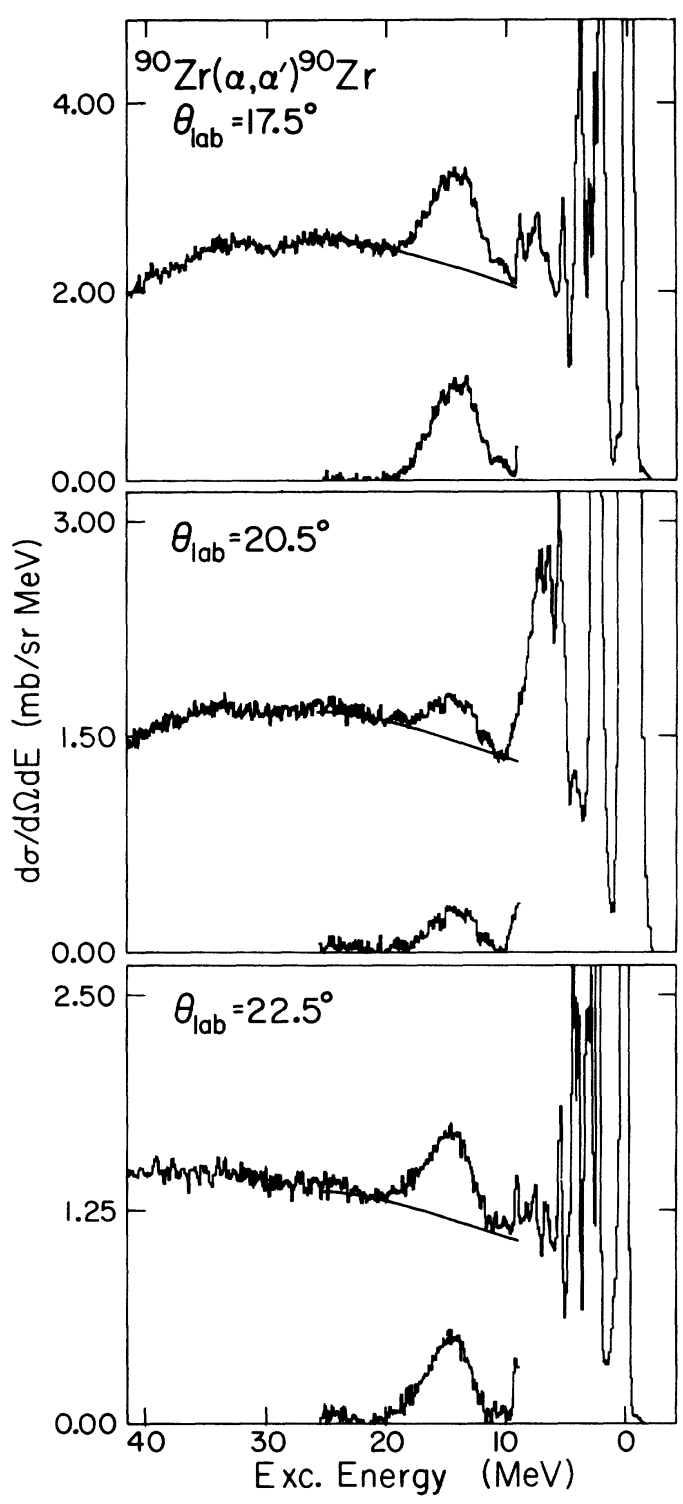

FIG. 5. Spectra of ${ }^{90} \mathrm{Zr}$ at two maxima and one minimum $\left(\theta_{\text {lab }}=20.5^{\circ}\right)$ of the GQR angular distribution. Also shown are the background lines, determined from the $20.5^{\circ}$ spectrum, and the GQR peaks after background subtraction. 
the data before determination of the GQR parameters. Since there is considerable variety in the shape and complexity of the GQR peaks, two different methods for analyzing the subtracted spectra were employed in order to obtain various moments of the peaks. The first was to evaluate directly the peak areas, centroids, and rms widths from the spectra with background subtracted. Each rms width was multiplied by 2.35 and is thus comparable to the FWHM of a Gaussian function. The second method was to fit the GQR with a single Gaussian or Lorentzian function. Positions and widths of the GQR obtained by direct evaluation of centroids and $\mathrm{rms}$ widths and those obtained from Gaussian and Lorentzian fits differed at most by 100-200 keV. The Lorentzian fits resulted in $20-30 \%$ increases in peak yield, however, due to the relatively large contributions in the tail regions. Since the data were generally fitted better by a Gaussian than by a Lorentzian, none of the quoted parameters have been derived from a Lorentzian fit. For many of the nuclei, the choice of analysis technique was dictated by
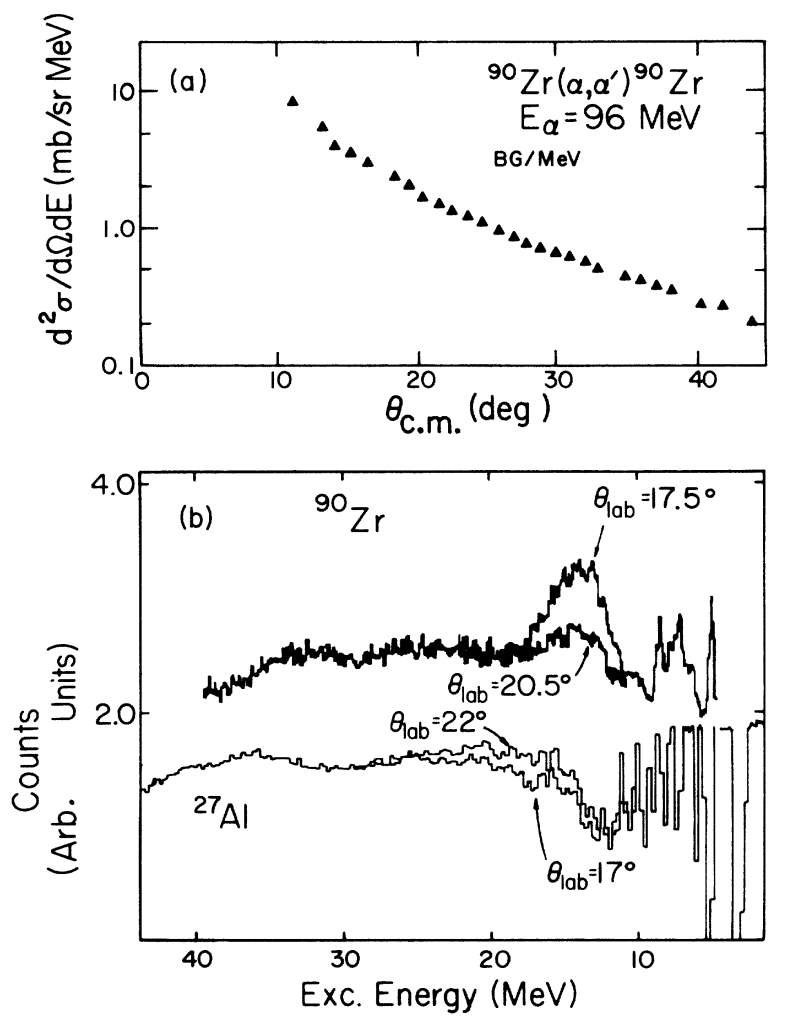

FIG. 6. (a) The angular distribution of the background just above the GQR peak for ${ }^{90} \mathrm{Zr}$. (b) ${ }^{90} \mathrm{Zr}$ (top) and ${ }^{27} \mathrm{Al}$ (bottom) spectra at two angles where the structure in the GQR region is different. For ${ }^{27} \mathrm{Al}$ some enhancement is apparent but there would be considerable ambiguity in choosing a background. the data itself. In ${ }^{56} \mathrm{Fe},{ }^{58} \mathrm{Ni}$, and ${ }^{59} \mathrm{Co}$ the GQR overlapped with a group of states at slightly lower excitation, which made it necessary to do a multiple-peak fit to the entire region to extract the parameters of the GQR. For nuclei where the peaks were not symmetric (all nuclei with $A \geqslant 141$ and several of the lighter nuclei), rms widths, centroids, and peak areas were obtained directly from the data. For the rare earth nuclei where spectra with high statistics are suggestive of a multiple-peak structure for the GQR, a two-peak analysis was also performed, the results of which are briefly discussed in Sec. VI.

Energies and widths obtained for the GQR peaks are listed in Table II, and angular distributions are shown in Fig. 7. The uncertainties given for the width determinations are rms deviations from the mean of the different spectra. Uncertainties due to energy calibration, background choice, etc., are considerably smaller. For nuclei where only a few spectra were available the uncertainties were estimated. Error bars given on the differential cross section measurements represent relative errors only and do not include allowances for significantly different background shapes. Using different background functions at different angles could change relative yields by $10-15 \%$ if the same criteria were used for all angles. Significantly different criteria for the background choice could alter peak yields by as much as $30-50 \%$.

The angular distributions of the GQRs for all the targets studied show diffraction patterns which are quite similar to those exhibited by the low-lying $2^{+}$states (Fig. 8), while the background underlying the GQR decreases monotonically with angle. Also, except in the case of ${ }^{208} \mathrm{~Pb}$, the peak shapes show little variation as a function of angle. These features suggest that no more than one or two multipoles are dominant in the excitation of this peak. In particular, the cross section of the GDR must be very small even in non-self-conjugate nuclei where isospin conservation does not forbid its excitation.

\section{DISTORTED WAVE BORN APPROXIMATION AND SUM RULES}

The distorted wave Born approximation (DWBA) transition amplitude for inelastically exciting a nucleus is given by

$$
T=\left\langle\chi_{f}^{(-)} \psi^{*}|\Delta U| \chi_{i}^{(+)} \psi\right\rangle,
$$

where $\chi_{i, f}$ represent the incoming and outgoing distorted waves and $\Delta U$ is the operator for the transition. The transition operators and energy weighted sum rules (EWSR) applicable to excitation of giant multipole resonances by inelastic 
TABLE II. Giant resonance parameters obtained from $\left(\alpha, \alpha^{\prime}\right)$. The number of spectra used to obtain $E$ and $\Gamma$ are indicated.

\begin{tabular}{|c|c|c|c|c|c|c|}
\hline Nucleus & $\begin{array}{c}E_{\text {exc }} \\
(\mathrm{MeV})\end{array}$ & $\begin{array}{c}\Gamma \\
(\mathrm{MeV})\end{array}$ & $\begin{array}{c}\% E 2 \\
\text { EWSR }\end{array}$ & $\beta^{2} R^{2}$ & $\begin{array}{c}\text { No. } \\
\text { spectra }\end{array}$ & $\begin{array}{c}\text { Type } \\
\text { analysis }^{a}\end{array}$ \\
\hline${ }^{32} \mathrm{~S}$ & $18.4 \pm 0.3$ & $7.1 \pm 0.5$ & $32 \pm 15$ & 0.46 & 8 & $R$ \\
\hline${ }^{36} \mathrm{Ar}$ & $18.3 \pm 0.3$ & $5.6 \pm 0.3$ & $49 \pm 15$ & 0.64 & 11 & $R$ \\
\hline${ }^{40} \mathrm{Ar}$ & $17.6 \pm 0.3$ & $4.7 \pm 0.3$ & $29 \pm 10$ & 0.36 & 8 & $R$ \\
\hline \multirow[t]{2}{*}{${ }^{40} \mathrm{Ca}{ }^{b}$} & $18.1 \pm 0.3$ & $3.5 \pm 0.3$ & $44 \pm 10$ & 0.53 & 11 & $R$ \\
\hline & $17.9 \pm 0.3$ & $3.4 \pm 0.3$ & $41 \pm 11$ & 0.50 & 7 & $R$ \\
\hline${ }^{48} \mathrm{Ti}$ & $18.0 \pm 0.5$ & $6.6 \pm 0.4$ & & & 3 & $G$ \\
\hline${ }^{56} \mathrm{Fe}$ & $16.7 \pm 0.3$ & $5.7 \pm 0.5$ & $49 \pm 15$ & 0.44 & 15 & $G$ \\
\hline${ }^{58} \mathrm{Ni}$ & $16.4 \pm 0.3$ & $4.9 \pm 0.2$ & $55 \pm 15$ & 0.50 & 10 & $G$ \\
\hline${ }^{59} \mathrm{Co}$ & $16.3 \pm 0.5$ & $5.6 \pm 0.4$ & & & 2 & $G$ \\
\hline${ }^{60} \mathrm{Ni}$ & $16.6 \pm 0.3$ & $5.0 \pm 0.4$ & $63 \pm 15$ & 0.55 & 8 & $\boldsymbol{R}$ \\
\hline${ }^{66} \mathrm{Zn}$ & $15.8 \pm 0.7$ & $5.8 \pm 0.8$ & & & 2 & $G$ \\
\hline${ }^{90} \mathrm{Zr}$ & $14.5 \pm 0.3$ & $4.0 \pm 0.2$ & $54 \pm 15$ & 0.37 & 8 & $R$ \\
\hline${ }^{94} \mathrm{Mo}$ & $14.4 \pm 0.4$ & $5.2 \pm 0.5$ & & & 4 & $G$ \\
\hline${ }^{96} \mathrm{Mo}$ & $14.4 \pm 0.6$ & $4.8 \pm 0.6$ & & & 2 & $G$ \\
\hline${ }^{100} \mathrm{Mo}$ & $13.6 \pm 0.4$ & $5.1 \pm 0.5$ & & & 5 & $G$ \\
\hline${ }^{141} \mathrm{Pr}$ & $13.3 \pm 0.4$ & $4.0 \pm 0.4$ & c & & 3 & $R$ \\
\hline${ }^{142} \mathrm{Nd}$ & $13.2 \pm 0.4$ & $3.6 \pm 0.3$ & $110 \pm 30^{d}$ & 0.51 & 7 & $R$ \\
\hline${ }^{144} \mathrm{Sm}$ & $13.0 \pm 0.3$ & $3.9 \pm 0.2$ & $91 \pm 25$ & 0.42 & 8 & $R$ \\
\hline${ }^{148} \mathrm{Sm}$ & $12.5 \pm 0.2$ & $4.3 \pm 0.2$ & $104 \pm 25$ & 0.49 & 12 & $R$ \\
\hline${ }^{154} \mathrm{Sm}$ & $12.4 \pm 0.3$ & $4.7 \pm 0.3$ & $102 \pm 25$ & 0.49 & 14 & $R$ \\
\hline${ }^{159} \mathrm{~Tb}$ & $12.0 \pm 0.6$ & $4.7 \pm 0.4$ & c & & 2 & $R$ \\
\hline${ }^{174} \mathrm{Yb}$ & $12.5 \pm 0.6$ & $4.7 \pm 0.5$ & c & & 15 & $R$ \\
\hline \multirow[t]{2}{*}{${ }^{208} \mathrm{~Pb}^{\mathrm{b}}$} & $10.8 \pm 0.4$ & $2.6 \pm 0.4$ & $93 \pm 25$ & 0.36 & 17 & $R$ \\
\hline & $10.6 \pm 0.4$ & $2.6 \pm 0.3$ & $92 \pm 25$ & 0.35 & 18 & $R$ \\
\hline
\end{tabular}

${ }^{\mathrm{a}} G=$ Gaussian fit; $R=$ centroid, rms width $\times 2.35$, integrated counts in region (no fit).

${ }^{\mathrm{b}}$ Upper row was obtained at $E_{\alpha}=96 \mathrm{MeV}$, lower row at $E_{\alpha}=115 \mathrm{MeV}$.

c Target thickness unknown due to nonuniformity.

d Target thickness determined by elastic yield relative to ${ }^{144} \mathrm{Sm}$.

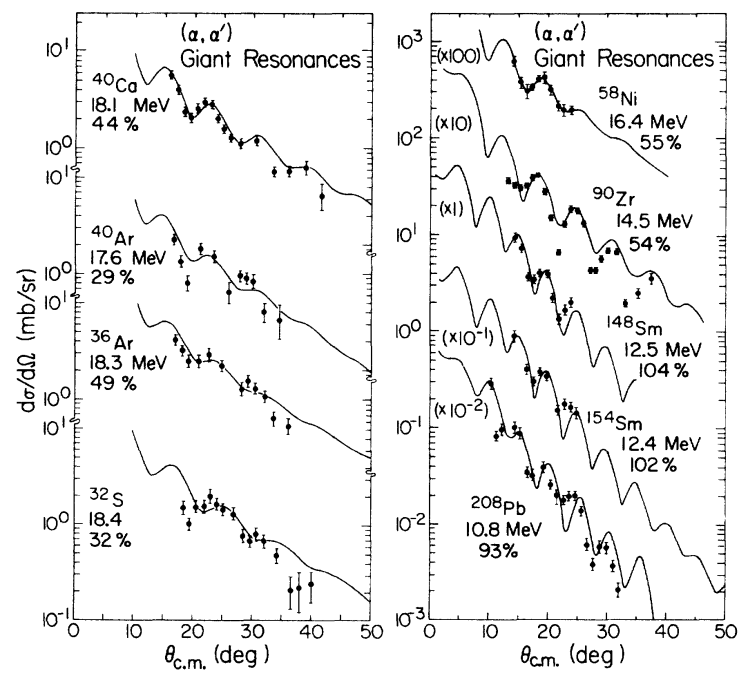

FIG. 7. Angular distributions obtained for the GQR peaks. The excitation energies and E2 EWSR percentages are indicated. The curves are DWBA calculations for an $L=2$ transfer. Error bars indicate relative errors only and do not allow for significantly different background shapes. The ${ }^{58} \mathrm{Ni}$ and ${ }^{148,154} \mathrm{Sm}$ data are for $E_{\alpha}=115 \mathrm{MeV}$. The rest are $E_{\alpha}=96 \mathrm{MeV}$.

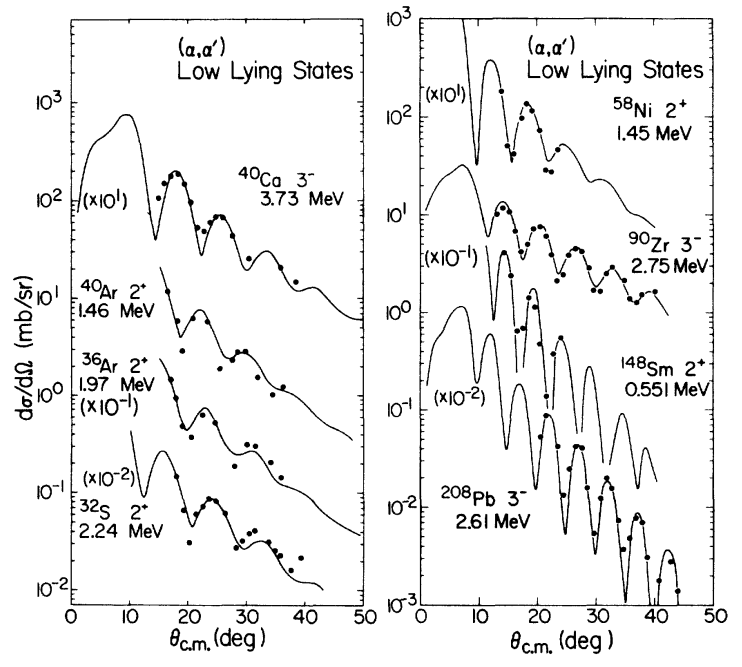

FIG. 8. Angular distributions obtained for low-lying states in several nuclei. The curves are DWBA calculations for the $L$ transfer indicated. 
scattering have been described in detail by Satchler. ${ }^{17}$ Those used in this study are summarized below for the different multipoles. In all EWSR determinations a uniform matter distribution was used to relate the deformation parameter $\beta$ to $B(E l)$ as well as to evaluate the moment $\left\langle r^{2 l-2}\right\rangle$ in the EWSR.

\section{A. Monopole (breathing mode) state}

For the excitation of a giant monopole resonance the transition operator is

$$
\Delta U=U_{1}(r) \beta_{0} Y_{00}(\Omega),
$$

where the form factor is (Satchler version II):

$$
U_{1}=-X U_{0}(r)-R \frac{d U_{0}(r)}{d r}
$$

with $X=\left[3+(\pi a / R)^{2}\right] /(1+\pi a / R)^{2} . \quad U_{0}=V+i W$ is the optical model potential utilized in the entrance and exit channels with radius and diffuseness parameters $R$ and $a$. For a monopole state located at an excitation energy $E_{x}$ which exhausts the EWSR,

$$
\beta_{0}^{2} R^{2}=\left(\frac{20 \pi}{3 A}\right) \frac{\hbar^{2}}{2 m E_{x}} .
$$

\section{B. Isovector dipole state}

For $\alpha$ particle excitation of a Goldhaber-Teller type GDR where the central neutron and proton densities are assumed to be equal

$$
\begin{array}{r}
\Delta U_{M}=(3)^{-1 / 2} U_{1}(r) \bar{d}_{1} Y_{1 M}(\Omega) \\
\text { with } \bar{d}_{1}=\sqrt{3}\left\langle 1 M\left|d_{1 M}^{+}\right| 00\right\rangle,
\end{array}
$$

where

$$
U_{1}(r)=\left(\frac{\pi}{3}\right)^{1 / 2}\left(\frac{N-Z}{A}\right)\left[\frac{\partial U_{0}(r)}{\partial r}+\frac{R}{3} \frac{\partial^{2} U_{0}(r)}{\partial r^{2}}\right] .
$$

The quantity $\bar{d}_{1}$ is proportional to the distance of separation of the proton and neutron distributions. For a GDR which exhausts the EWSR,

$$
\bar{d}_{1}^{2}=\frac{\hbar^{2}}{2 m E_{x}} \frac{3 A}{N Z} \text { 。 }
$$

\section{Isoscalar quadrupole and higher multipoles}

The transition operator for $L=2$ and higher multipoles is

$$
\Delta U_{L M}=-\beta_{L} U_{1}(r) Y_{L M}(\Omega)(2 l+1)^{-1 / 2},
$$

with $U_{1}(r)=R \partial U_{0}(r) / \partial r$. For a state which exhausts the EWSR, $\beta_{L}{ }^{2} R^{2}=l(2 l+1)\left(\hbar^{2} / 2 m E_{x}\right)(4 \pi / 3 A)$.

The experimental and DWBA cross sections are related by $d \sigma_{\exp } / d \sigma_{\text {DWBA }}=\beta^{2}$. Since $\alpha$ scattering is sensitive to the shape of the matter distribution rather than the charge distribution, the EWSR strengths derived here are comparable to electromagnetic measurements if $(\beta)_{\text {matter }}=(\beta)_{\text {protor }}$

Computer codes were written to generate the monopole and dipole form factors which were then read into DWUCK ${ }^{18}$ for DWBA calculations. Cases corresponding to those shown by Satchler ${ }^{17}$ were run to check the computer codes. Calculations for $L=2$ and $L=4$ were performed with available options in DWUCK.

\section{COMPARISON OF THE DWBA CALCULATIONS WITH THE DATA}

The DWBA calculations utilized optical potentials (Table III) derived from elastic scattering data taken along with the inelastic data. The use of quite different optical model parameter sets for ${ }^{90} \mathrm{Zr}$ resulted in little difference in the quality of the fits and at most a $10 \%$ variation in $\beta^{2} R^{2}$. Using different parameters for incoming and outgoing channels had a similar effect. The insensitivity of DWBA predictions for the $\left(\alpha, \alpha^{\prime}\right)$ reaction to optical parameter ambiguities has been well established. ${ }^{14}$ Using the derived potentials, the differential cross sections for the low-lying collective states are well reproduced (Fig. 8) and $B(E 2)$ values obtained are in agreement with those deduced from other techniques (Table IV).

DWBA predictions for $L=0$ (breathing mode) and $L=1$ (isovector dipole) transitions exhausting $100 \%$ of the EWSR as well as predictions for $L=2$ and $L=4$ excitations normalized to the data are shown for the GQRs in ${ }^{40} \mathrm{Ca},{ }^{90} \mathrm{Zr}$, and ${ }^{208} \mathrm{~Pb}$ in Fig. 9 . The prediction for the monopole state is slightly out of phase with the data and about a factor of 3 too weak to account for the observed strength. Unfortunately, no breathing mode state is known which can be used to test the form factor. Bertsch and $\mathrm{Tsai}^{21}$ have demonstrated, however, that sim-

TABLE III. Optical potentials used for DWBA calculations.

\begin{tabular}{cccccc}
\hline \hline Nucleus & $\begin{array}{c}E_{\alpha} \\
(\mathrm{MeV})\end{array}$ & $\begin{array}{c}V \\
(\mathrm{MeV})\end{array}$ & $\begin{array}{c}W \\
(\mathrm{MeV})\end{array}$ & $\begin{array}{c}r_{0} \\
(\mathrm{fm})\end{array}$ & $\begin{array}{c}a \\
(\mathrm{fm})\end{array}$ \\
\hline${ }^{32} \mathrm{~S}$ & 96 & 75.2 & 46.8 & 1.36 & 0.76 \\
${ }^{36} \mathrm{Ar}$ & 96 & 104.4 & 56.5 & 1.24 & 0.85 \\
${ }^{40} \mathrm{Ar}$ & 96 & 120.0 & 68.6 & 1.20 & 0.85 \\
${ }^{40} \mathrm{Ca}$ & 96 & 121.9 & 62.7 & 1.35 & 0.70 \\
& 115 & 109.7 & 61.5 & 1.35 & 0.70 \\
${ }^{58} \mathrm{Ni}$ & 115 & 78.9 & 38.0 & 1.28 & 0.90 \\
${ }^{90} \mathrm{Zr}$ & 96 & 45.7 & 27.7 & 1.50 & 0.70 \\
${ }^{148} \mathrm{Sm}$ & 115 & 59.3 & 40.5 & 1.39 & 0.79 \\
${ }^{208} \mathrm{~Pb}$ & 96 & 76.2 & 51.3 & 1.39 & 0.66 \\
& 115 & 89.3 & 52.7 & 1.35 & 0.71 \\
\hline \hline
\end{tabular}

${ }^{a}$ Reference 10. 
TABLE IV. Transition probabilities obtained for low-lying collective states. $B(E L)$ values were obtained in the manner described by Bernstein (Ref. 14) using a Fermi matter distribution with $R_{\text {matter }}=\left(1.2 \mathrm{~A}^{1 / 3}\right) \mathrm{fm}$.

\begin{tabular}{ccccccc}
\hline \hline & \multirow{2}{*}{$E_{\text {exc }}$} & \multicolumn{3}{c}{ Present work } & \multicolumn{2}{c}{ Other work } \\
Nucleus & $(\mathrm{MeV})$ & $J^{\pi}$ & $\beta R$ & $B(E L)$ & $B(E L)(E M)$ & $B(E L)\left(\alpha, \alpha^{\prime}\right)$ \\
\hline${ }^{32} \mathrm{~S}$ & 2.24 & $2^{+}$ & 1.16 & $11.5 \pm 2.0$ & $8.8 \pm 1.5^{\mathrm{a}}$ & \\
${ }^{36} \mathrm{Ar}$ & 1.97 & $2^{+}$ & 0.88 & $7.5 \pm 2.0$ & $9.4 \pm 1.0^{\mathrm{a}}$ & \\
${ }^{40} \mathrm{Ar}$ & 1.46 & $2^{+}$ & 0.87 & $6.7 \pm 2.0$ & $12.5 \pm 3.0^{\mathrm{a}}$ & \\
${ }^{40} \mathrm{Ca}$ & 3.73 & $3^{-}$ & 1.12 & $18.6 \pm 3.0$ & $31.6 \pm 4.7^{\mathrm{b}}$ & $23.6 \pm 3.5^{\mathrm{b}}$ \\
${ }^{58} \mathrm{Ni}$ & 1.45 & $2^{+}$ & 0.94 & $13.7 \pm 2.0$ & $9.9 \pm 1.5^{\mathrm{b}}$ & $15.8 \pm 1.6^{\mathrm{b}}$ \\
${ }^{90} \mathrm{Zr}$ & 2.75 & $3^{-}$ & 0.79 & $16.4 \pm 2.0$ & $32 \pm 5^{\mathrm{b}}$ & $20.0 \pm 1.6^{\mathrm{b}}$ \\
${ }^{148} \mathrm{Sm}$ & 0.55 & $2^{+}$ & 0.94 & $34 \quad \pm 5$ & $38.5^{\mathrm{c}}$ & \\
${ }^{208} \mathrm{~Pb}$ & 2.61 & $3^{-}$ & 0.82 & $36 \pm 5$ & $39.5 \pm 2.2^{\mathrm{b}}$ & $35.8 \pm 3.6^{\mathrm{b}}$ \\
\hline \hline
\end{tabular}

${ }^{a}$ Reference 19.

${ }^{\mathrm{b}}$ Reference 14 .

${ }^{\mathrm{c}}$ Reference 20 .

ilar monopole form factors are obtained with microscopic calculations. The excitation of the isovector dipole is isospin forbidden in ${ }^{40} \mathrm{Ca}$ and predicted to be about a factor of 5 weaker than the observed peak strength in ${ }^{90} \mathrm{Zr}$ and ${ }^{208} \mathrm{~Pb}$. The predicted dipole angular distribution is out of phase with the observed distribution, so that the GDR would contribute more to the peak in the minima and would tend to wash out the diffraction structure in the angular distribution. The assumption (in this calculation) that all of the neutron excess is confined to the surface is not realistic and more reasonable assumptions would reduce the predicted GDR cross section. Consistent with these predictions, no evidence for excitation of the GDR was seen in any of the nuclei.

The data are best fitted generally by an $L=2$ calculation (see Figs. 7 and 9) with both the phase and general shape of the data well reproduced. The deduced strength varies from $32-110 \%$ of the E2 EWSR. Although the phase of the GQR peaks and the low-lying $2^{+}$states are very nearly the same for all nuclei studied, the DWBA predicts a $0.5^{\circ}$ to $1.5^{\circ}$ shift in phase which, for heavy nuclei, results in a relatively poor $L=2$ fit to the GQR. In fact, for nuclei with $A \gtrsim 141$ DWBA calculations for $L=4$ transfer fitted the data as well as $L=2$ calculations. Only $10-25 \%$ of the $L=4$ EWSR strength is required to reproduce the observed cross section; thus small components of $L=4$ strength could contribute significantly. There is good agreement (see Sec. VII) between the present work and $L=2$ strength located by electron scattering (where $L=4$ can be easily distinguished), suggesting that the peak in our data is primarily due to $L=2$ strength. This suggests that the $Q$ dependence predicted by the DWBA is not correct for these highly excited states.

\section{DISCUSSION}

For all nuclei studied with $A \geqslant 36$ a clustering of a significant fraction of the E2 strength into one broad peak is observed. The E2 EWSR fractions and peak widths observed are given in Table II. In the lighter nuclei $32-60 \%$ of the E2 strength is contained in a peak of $3.5-7.1 \mathrm{MeV}$ width, with the strength increasing in heavier nuclei until $90-110 \%$ is seen for $A \approx 141-208$ in peaks of width 2.6-4.7 MeV. Peak parameters obtained at 96 $\mathrm{MeV}$ are in excellent agreement with those obtained at $115 \mathrm{MeV}$. In nuclei lighter than $A=36$ our data show that the GQR peak has weakened and/or broadened to such an extent that it is no longer a prominent feature of the spectrum. Assuming a Gaussian peak shape, $S / \Gamma$ (where $S$ is the percentage of the E2 EWSR) must be less than $\sim 3.5 \% / \mathrm{MeV}$ for these nuclei. ${ }^{15}$

Several indications of substructure are apparent in the GQR region. In ${ }^{36} \mathrm{Ar},{ }^{40} \mathrm{Ca},{ }^{56} \mathrm{Fe},{ }^{58} \mathrm{Ni}$, and ${ }^{59} \mathrm{Co}$ there is a weaker peak 3-4 MeV lower in excitation energy, having a width of approximately $1 \mathrm{MeV}$, which is not entirely resolved from the GQR. The lower peak in ${ }^{40} \mathrm{Ca}\left(E_{x}=14.1 \mathrm{MeV}\right)$ is fitted by an $L=2$ angular distribution exhausting $7 \%$ of the E2 EWSR. No meaningful angular distributions were obtained for the other nuclei. In ${ }^{56} \mathrm{Fe}\left(E_{x}=13.3 \mathrm{MeV}\right)$ and ${ }^{58} \mathrm{Ni}\left(E_{x}=13.3 \mathrm{MeV}\right)$ there are indications of considerable structure in this peak. This structure changes with angle however, indicating the presence of several multipolarities. In ${ }^{90} \mathrm{Zr}$ the GQR peak is nearly symmetric and within statistics has no substructure. All nuclei studied in the mass region $A=141-174$ exhibit a GQR which is quite asymmetric with considerable tailing on the high excitation side and contains $\sim 100 \%$ of the E2 EWSR. The GQR for the $N=82$ 
nuclei are quite suggestive of two unresolved peaks separated by $\sim 2.5 \mathrm{MeV}$. A ${ }^{144} \mathrm{Sm}$ spectrum, after background subtraction, is shown with a two-peak fit superimposed in Fig. 10. A fit with two peaks was also made to the ${ }^{148} \mathrm{Sm}$ data where more angles with relatively good statistics were available. Angular distributions for both components, shown in Fig. 11, are best fitted by $L=2$ DWBA calculations.

Rather complete measurements were obtained for ${ }^{208} \mathrm{~Pb}$ at 96 and $115 \mathrm{MeV}$ and several interest-

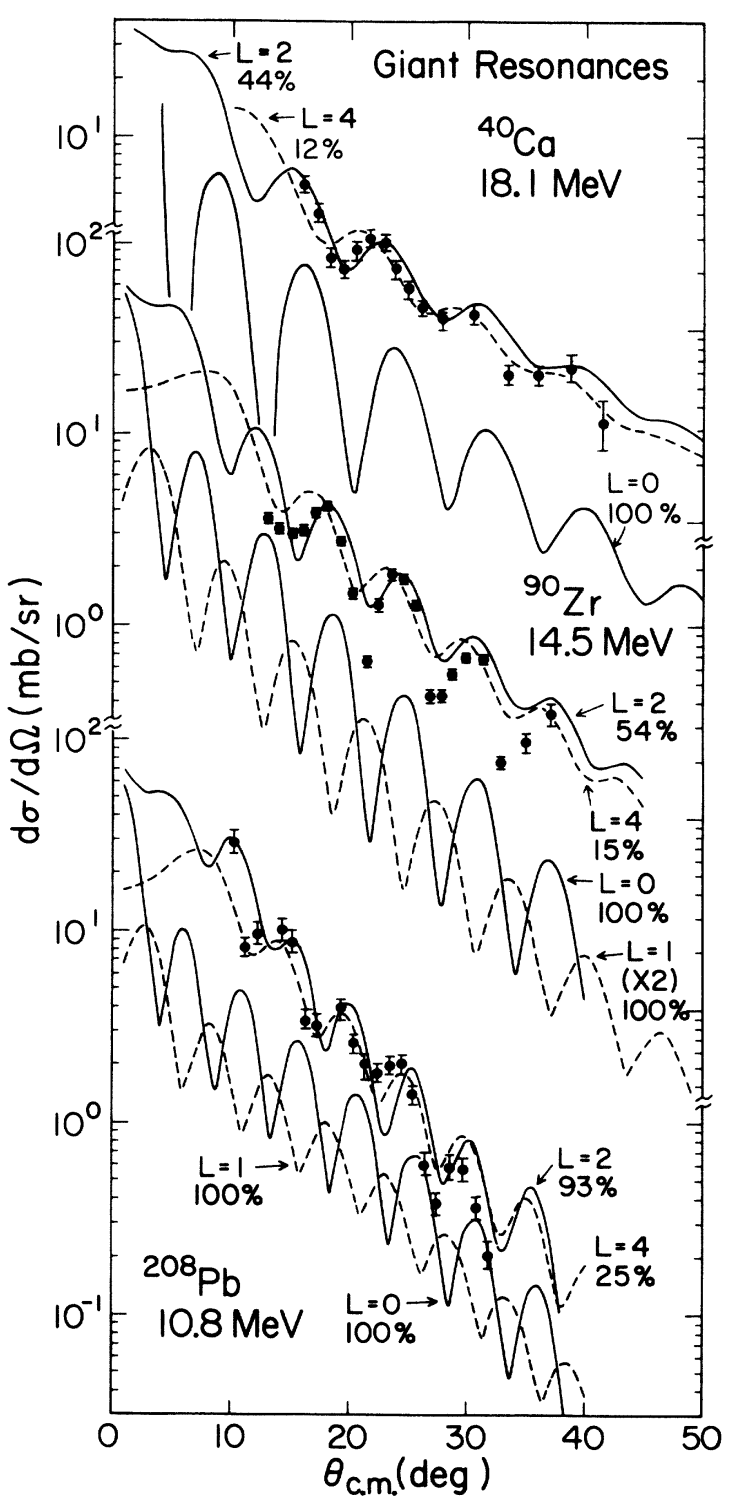

FIG. 9. DWBA calculations for monopole, isovector dipole, quadrupole and hexadecapole transitions in ${ }^{40} \mathrm{Ca}$, ${ }^{90} \mathrm{Zr}$, and ${ }^{208} \mathrm{~Pb}$ shown with the experimental data taken at $96 \mathrm{MeV}$. Excitation of the dipole is isospin forbidden in ${ }^{40} \mathrm{Ca}$. The sum-rule percentage for each calculation is shown.

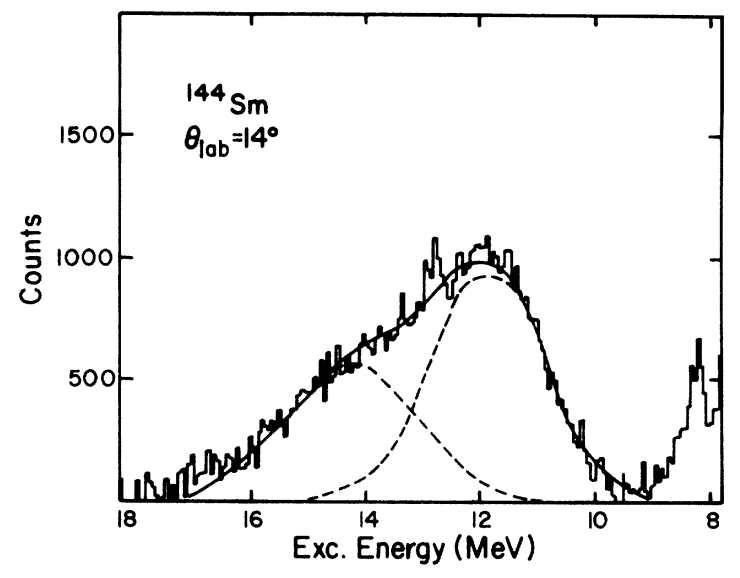

FIG. 10. The GQR of ${ }^{144} \mathrm{Sm}$ after background subtraction. A two-peak Gaussian fit is shown superimposed.

ing features have emerged. Narrow peaks at 8.6 and $9.1 \mathrm{MeV}$ are superimposed on a broad peak $(\Gamma=2.6 \mathrm{MeV})$ at $10.7 \mathrm{MeV}$. The yield of the broad peak would correspond to $\sim 93 \%$ of the E2 EWSR strength; however, the angular distributions are fitted as well by $L=4$ as $L=2$, not inconsistent with the calculation of Halbert et al. ${ }^{22}$ which predicts significant $L=4$ strength in the peak. The shape of the GQR peak changes somewhat with angle for ${ }^{208} \mathrm{~Pb}$ suggesting the presence of more than one multipole. Some evidence is seen for the multiplet structure observed in the broad peak

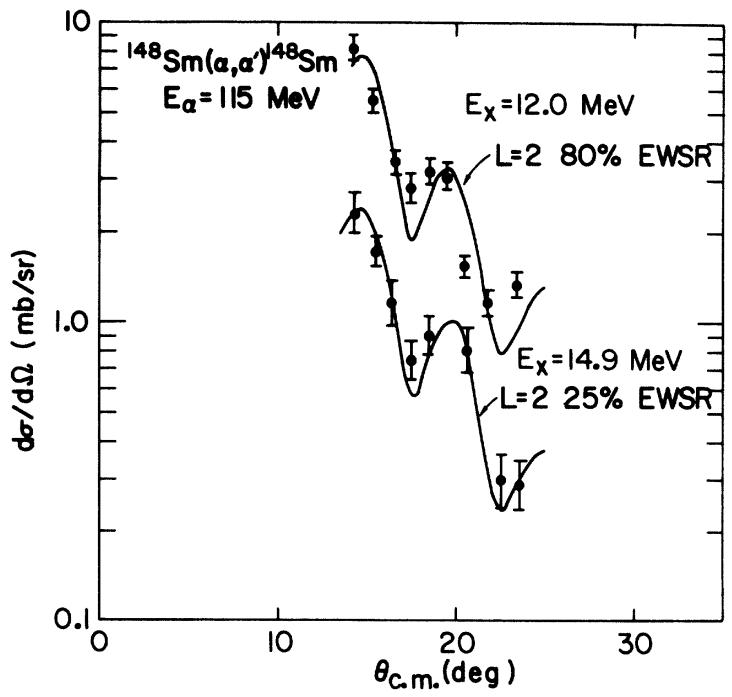

FIG. 11. Angular distribution for each peak of the doublet fitted to the GQR in ${ }^{148} \mathrm{Sm}$. The curves are $L$ $=2$ DWBA predictions. The error bars indicate relative errors only and do not allow for significantly different background shapes. 


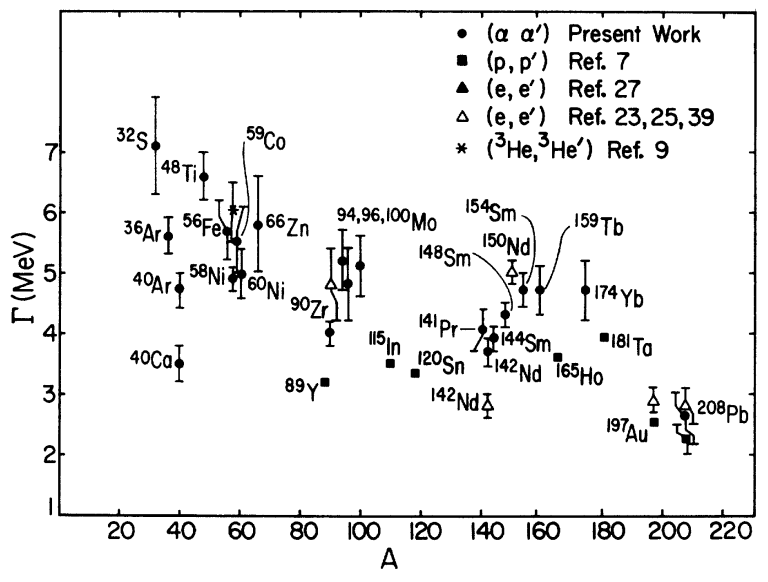

FIG. 12. The full width at half-maximum of the GQR peaks obtained in a number of nuclei are plotted vs $A$.

with electron scattering. ${ }^{23-25}$ The secondary peak at $8.6 \mathrm{MeV}$ has a width of $\sim 200 \mathrm{keV}$. Due to the large background and weak contaminants present, a good angular distribution could not be obtained for the $8.6 \mathrm{MeV}$ peak. The angular distribution of the peak at $9.1 \mathrm{MeV}$ is out of phase with the GQR, suggesting an odd multipolarity。

The general trend of the widths may be seen in Fig. 12. The GQR peaks are narrowest for closed

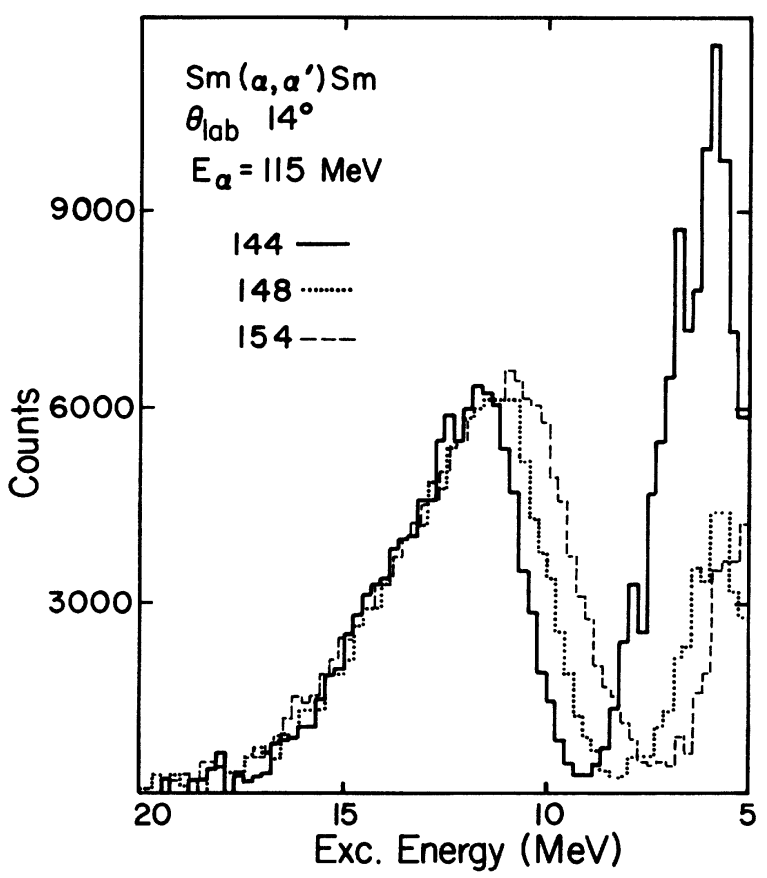

FIG. 13. The ${ }^{144,148,154} \mathrm{Sm}$ GQR peaks are shown superimposed. Background has been subtracted and the peaks have been normalized to the same height. shell nuclei $\left({ }^{40} \mathrm{Ca},{ }^{90} \mathrm{Zr},{ }^{144} \mathrm{Sm},{ }^{208} \mathrm{~Pb}\right)$ and the width decreases as $A$ increases, reminiscent of the behavior of the GDR. A comparison of the GQR peaks for ${ }^{144,148,154} \mathrm{Sm}$ are shown in Fig. 13. It is apparent that the width increases as one goes from the spherical to the soft to the deformed nuclei, with $\Gamma_{154}-\Gamma_{148} \approx \Gamma_{148}-\Gamma_{144} \approx 0.4 \mathrm{MeV}$. Thus, permanently deformed ${ }^{154} \mathrm{Sm}$ has a width $0.8 \pm 0.3$ $\mathrm{MeV}$ greater than spherical ${ }^{144} \mathrm{Sm}$. Utilizing the usual $Q Q$ interaction, a $6 \mathrm{MeV}$ splitting of the GQR is predicted for ${ }^{15} \mathrm{Sm}$ due to the deformation; however, a rigorous application ${ }^{26}$ of self-consistency to the coupling of the quadrupole mode leads to a modified $Q Q$ interaction which reduces the splitting to about $2 \mathrm{MeV}$, consistent with the observed broadening.

\section{COMPARISON WITH OTHER WORK}

In order to assess the reliability of the interpretation of the data, the many different reactions populating GQRs must be compared. Figure 14 shows a comparison of one of our ${ }^{90} \mathrm{Zr}\left(\alpha, \alpha^{\prime}\right)$ spectra with an electron scattering spectrum taken by Fukuda and Torizuka ${ }^{27}$ at a momentum transfer where the $L=1$ contribution is weak compared to the $L=2$ (or $L=0$ ). The spectra are essentially identical in the giant resonance region (except for the small $E 1$ contribution) and yield similar values for excitation energy $(14.0 ; 14.5$ $\pm 0.3)$ sum-rule fraction $(56 \% ; 54 \%)$, and width $(4.8 \pm 0.6 ; 4.0 \pm 0.2)$. Figure 15 compares our ${ }^{58} \mathrm{Ni}\left(\alpha, \alpha^{\prime}\right)$ with the ${ }^{58} \mathrm{Ni}\left(d, d^{\prime}\right)$ of Chang et al. ${ }^{8}$ The spectra in the giant resonance region are again virtually identical, there being good agreement with both the big peak at $16.4 \mathrm{MeV}$ and the smaller (unresolved) peak at $13.3 \mathrm{MeV}$. Both of these reactions should excite primarily isoscalar strength.

A comparison of giant resonance parameters ex-

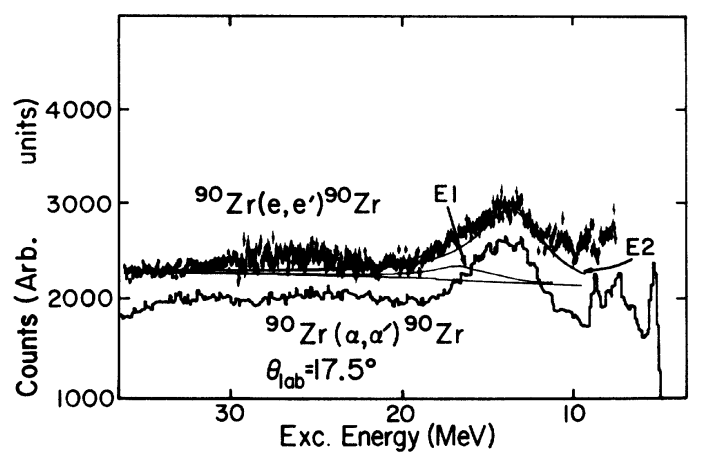

FIG. 14. The lower spectrum is ${ }^{90} \mathrm{Zr}\left(\alpha, \alpha^{\prime}\right)$ at $96 \mathrm{MeV}$. The upper is ${ }^{90} \mathrm{Zr}\left(e, e^{\prime}\right)$ from Ref. 27. The lines are the $E 2$ (large peak) and $E 1$ (small peak) components, respectively of the $\left(e, e^{\prime}\right)$ data. 
tracted by different reactions is given in Table $\mathrm{V}$. Inelastic proton and $\alpha$ scattering on ${ }^{40} \mathrm{Ca}$ indicate a center of the quadrupole strength at $\sim 18 \mathrm{MeV}$ containing $\sim 45 \%$ of the EWSR. From electron scattering Torizuka et al. ${ }^{28}$ deduce a total quadrupole strength of about $37 \%$ between $16-22 \mathrm{MeV}$, with $\sim 29 \%$ between $10-16 \mathrm{MeV}$. For ${ }^{58} \mathrm{Ni}$ the positions and shapes of the GQR obtained by $d$ and $\alpha$ inelastic scattering are almost identical, and EWSR fractions agree within the errors (primarily background subtraction uncertainties). Inelastic electron and $\alpha$ scattering for ${ }^{90} \mathrm{Zr}$ yield similar values for the energy, width and sum rule fraction for the GQR. For ${ }^{144} \mathrm{Sm},{ }^{154} \mathrm{Sm}$, and ${ }^{208} \mathrm{~Pb}$ the inelastic ${ }^{3} \mathrm{He}$ scattering results ${ }^{9}$ are in disagreement with the other data. The reported peak widths and sum rule fractions are consistently much larger than other results. A comparison of the ${ }^{3} \mathrm{He}$ and $\alpha$ spectra for these nuclei clearly indicates the source of the problem. In ${ }^{3} \mathrm{He}$ scattering the yield increases almost uniformly from the edge of the spectra ( $48 \mathrm{MeV}$ excitation) to the region of the GQR where it drops abruptly and discrete states become prominent, whereas for $\alpha$ scattering there is a prominent peak rising above the background. The authors of Ref. 9 discuss the problems of analyzing the ${ }^{3} \mathrm{He}$ data, pointing out that the shapes of the spectra are very similar for all of the nuclei studied and hence similar parameters are obtained. A comparison with both $\alpha$ and electron data would indicate that they may have included a large amount of the background in their "GQR peak."

For ${ }^{208} \mathrm{~Pb}$ there are several inconsistencies between different experiments. Except for the ${ }^{3} \mathrm{He}$ data all obtain widths of 2.5-3.0 MeV for the GQR. Fine structure seen in electron ${ }^{23-25}$ and proton ${ }^{6,7,30}$ scattering superimposed on the GQR is not as prominent in $\alpha$ scattering. The sum rule fractions obtained fall into two groups, one around $40 \%$, the

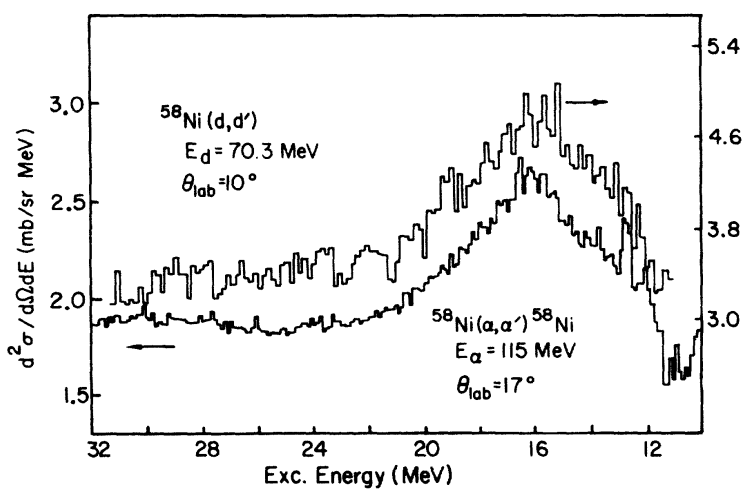

FIG. 15. The upper spectrum is ${ }^{58} \mathrm{Ni}\left(d, d^{\prime}\right)$ from Ref. 8 , the lower is ${ }^{58} \mathrm{Ni}\left(\alpha, \alpha^{\prime}\right)$ at $115 \mathrm{MeV}$. other about $100 \%$. With inelastic electron scattering Buskirk et al. ${ }^{23}$ reported a value of $37 \pm 13 \%$ for the strength, and Nagao and Torizuka ${ }^{24} \mathrm{ob}-$ tained $39 \pm 5 \%$. On the basis of a later experiment, Pitthan et $a l .{ }^{25}$ report a value of $95 \pm 35 \%$. The higher value is obtained ${ }^{31}$ by fitting the observed peak with a Lorentzian and calculating the area, whereas the smaller value results from adding observed counts over a limited region of the peak. Values obtained with hadron scattering include $39 \%\left[\left(p, p^{\prime}\right)\right.$ at $\left.155 \mathrm{MeV}\right],{ }^{7} 90 \%\left[\left(p, p^{\prime}\right)\right.$ at $\left.60 \mathrm{MeV}\right],{ }^{30}$ 93\% $\left[\left(\alpha, \alpha^{\prime}\right)\right.$, present work $\left.)\right], 120 \%\left[\left({ }^{3} \mathrm{He},{ }^{3} \mathrm{He}^{\prime}\right)\right],{ }^{32}$ and $165 \%\left[\left({ }^{3} \mathrm{He},{ }^{3} \mathrm{He}^{\prime}\right)\right] .{ }^{9}$ The ${ }^{3} \mathrm{He}$ results have large uncertainties due to background problems as discussed previously, while strengths obtained from the $155 \mathrm{MeV}\left(p, p^{\prime}\right)$ analysis are consistently lower than those from other reactions.

Comparisons of available widths and strengths for the GQR in many nuclei are shown in Figs。12 and 16 , respectively. Several features are readily apparent. There is in general good agreement between $\left(e, e^{\prime}\right)$ and $\left(\alpha, \alpha^{\prime}\right)$ results, both for specific values as well as over-all trends. The lower energy $\left(p, p^{\prime}\right)$ data and $\left(d, d^{\prime}\right)$ data also yield similar results. Both the sum rule fractions and widths obtained from $155 \mathrm{MeV}$ inelastic proton scattering by Marty et $a l_{\circ}{ }^{7}$ fall well below the general trends established from the other data.

Caution must be exercised when considering strengths relative to sum rules, however. Halbert et $a l .^{22}$ discuss thoroughly the problems involved in applying the sum rules to the several different types of experiments which have been performed. In addition to the uncertainties in relating $B(E 2)$ values from different experiments due to differing operators, they point out that if the isospin of the

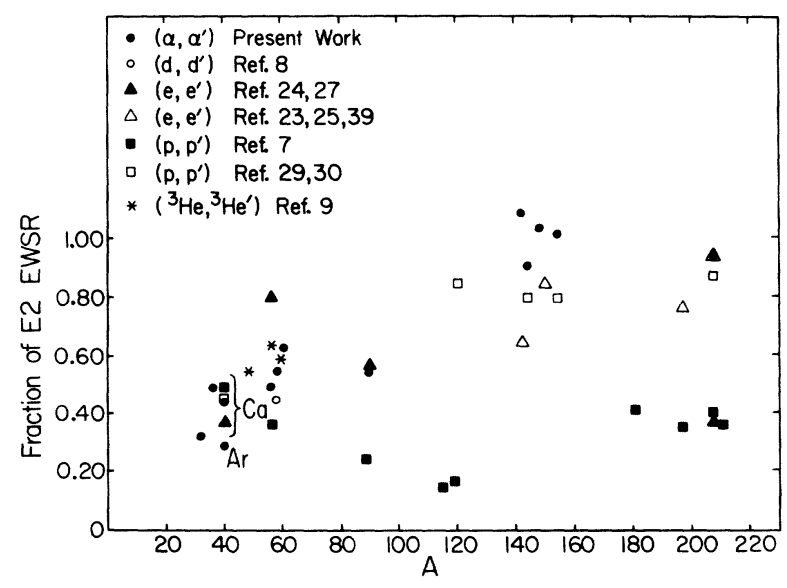

FIG. 16. EWSR fractions obtained for the GQR from various reactions are shown as a function of $A$. Error bars are omitted for clarity, but in no case are the errors smaller than $\pm 15 \%$. 
TABLE V. Comparison of giant resonance parameters obtained from various reactions. The results are those quoted by the authors.

\begin{tabular}{|c|c|c|c|c|c|}
\hline Nucleus & $\begin{array}{c}E_{\mathrm{exc}} \\
(\mathrm{MeV})\end{array}$ & $\begin{array}{c}\Gamma \\
(\mathrm{MeV})\end{array}$ & $\% S R$ & Reaction & Source \\
\hline \multirow[t]{4}{*}{${ }^{40} \mathrm{Ca}$} & $18.0 \pm 0.3$ & $3.5 \pm 0.3$ & $43 \pm 10$ & $\left(\alpha, \alpha^{\prime}\right)$ & Present work \\
\hline & $18.0 \pm 0.3$ & & $49 \pm 10$ & $\left(p, p^{\prime}\right)$ & Ref. 7 \\
\hline & $18.0 \pm 0.4$ & & $45 \pm 10$ & $\left(p, p^{\prime}\right)$ & Ref. 30 \\
\hline & $16-22$ & & 37 & $\left(e, e^{\prime}\right)$ & Ref. 28 \\
\hline \multirow[t]{2}{*}{${ }^{58} \mathrm{Ni}$} & $16.4 \pm 0.3$ & $4.9 \pm 0.2$ & $55 \pm 15$ & $\left(\alpha, \alpha^{\prime}\right)$ & Present work \\
\hline & $16.0 \pm 0.5$ & & $45 \pm 10$ & $\left(d, d^{\prime}\right)$ & Ref. 8 \\
\hline \multirow[t]{2}{*}{${ }^{90} \mathrm{Zr}$} & $14.5 \pm 0.3$ & $4.0 \pm 0.2$ & $54 \pm 15$ & $\left(\alpha, \alpha^{\prime}\right)$ & Present work \\
\hline & 14.0 & $4.8 \pm 0.6$ & $56 \pm 17$ & $\left(e, e^{\prime}\right)$ & Ref. 27 \\
\hline \multirow[t]{3}{*}{${ }^{144} \mathrm{Sm}$} & $13.0 \pm 0.3$ & $3.9 \pm 0.2$ & $91 \pm 25$ & $\left(\alpha, \alpha^{\prime}\right)$ & Present work \\
\hline & $12.8 \pm 0.3$ & & $80 \pm 20$ & $\left(p, p^{\prime}\right)$ & Ref. 29 \\
\hline & $13.0 \pm 0.5$ & 5.4 & $130 \pm 52^{a}$ & $\left({ }^{3} \mathrm{He},{ }^{3} \mathrm{He}^{\prime}\right)$ & Ref. 9 \\
\hline \multirow[t]{3}{*}{${ }^{154} \mathrm{Sm}$} & $12.4 \pm 0.3$ & $4.7 \pm 0.3$ & $102 \pm 25$ & $\left(\alpha, \alpha^{\prime}\right)$ & Present work \\
\hline & $12.5 \pm 0.3$ & & $80 \pm 20$ & $\left(p, p^{\prime}\right)$ & Ref. 30 \\
\hline & $12.2 \pm 0.5$ & 5.5 & $150 \pm 60^{\mathrm{a}}$ & $\left({ }^{3} \mathrm{He},{ }^{3} \mathrm{He}^{\prime}\right)$ & Ref. 9 \\
\hline \multirow[t]{8}{*}{${ }^{208} \mathrm{~Pb}$} & $10.7 \pm 0.4$ & $2.6 \pm 0.4$ & $93 \pm 25$ & $\left(\alpha, \alpha^{\prime}\right)$ & Present work \\
\hline & $\sim 10.4$ & & $39 \pm 5^{b}$ & $\left(e, e^{\prime}\right)$ & Ref. 24 \\
\hline & 10.5 & $2.8 \pm 0.3$ & $95 \pm 35$ & $\left(e, e^{\prime}\right)$ & Ref. 25 \\
\hline & $\sim 10.4$ & & $37 \pm 13$ & $\left(e, e^{\prime}\right)$ & Ref. 23 \\
\hline & 11 & $\sim 3$ & 120 & $\left({ }^{3} \mathrm{He},{ }^{3} \mathrm{He}^{\prime}\right)$ & Ref. 32 \\
\hline & $10.9 \pm 0.5$ & 5.9 & $165 \pm 66^{\mathrm{a}}$ & $\left({ }^{3} \mathrm{He}^{3} \mathrm{He}^{\prime}\right)$ & Ref. 9 \\
\hline & $10.7 \pm 0.3$ & & $90 \pm 15$ & $\left(p, p^{\prime}\right)$ & Ref. 30 \\
\hline & $9.2,10.8$ & $2-2.5$ & $39 \pm 8^{c}$ & $\left(p, p^{\prime}\right)$ & Ref. 7 \\
\hline
\end{tabular}

\footnotetext{
${ }^{a}$ The authors of Ref. 9 assign a $40 \%$ uncertainty to their peak cross section. This uncertainty has been assigned to their sum-rule (SR) percentage.

${ }^{\mathrm{b}}$ For comparative purposes only $E 2$ strength observed between $9.1-11.5 \mathrm{MeV}$ has been included. The contribution from the $8.6 \mathrm{MeV}$ state was omitted.

c The \% SR value listed here is the sum of the values given for the 9.2 and $10.8 \mathrm{MeV}$ peaks.
}

various components of the GQR is not pure, quite different results can be obtained with different projectiles. The relatively good agreement between the many experimental results may be an indication that the collective model (which relates inelastic scattering results to $B(E 2)$ values) is better than predicted by the calculations of Halbert $e t$ al.

There is now evidence from many reactions that the peak at $\sim 63 / A^{1 / 3} \mathrm{MeV}$ is predominantly $L=2$. Electron scattering experiments ${ }^{5,23-25}$ have shown for several nuclei that the peak is either $L=2$ or $L=0$. Angular distributions for inelastic hadron scattering, ${ }^{6-9}$ including the present work, favor $L=2$ over $L=0$ in most cases due both to the shape of the angular distributions and the magnitude of the cross section. Kocher et al. ${ }^{33}$ have looked at the inelastic scattering of polarized protons from ${ }^{58} \mathrm{Ni}$, but have not been able to make a definitive assignment.

For nuclei lighter than $A \approx 36$, our data indicate that a qualitative change occurs in the GQR. The E2 strength is no longer clustered in a single broad peak but must be fragmented among many levels over a wide energy range. This is consistent with several studies of nuclei in this re- gion. Singh and Yang ${ }^{34}$ have identified $50 \%$ of the isoscalar E2 strength in ${ }^{24} \mathrm{Mg}$ in discrete states below $18 \mathrm{MeV}$ by careful analysis of ${ }^{24} \mathrm{Mg}\left(\alpha, \alpha^{\prime}\right)$ data. Recently, data of Knöpfle et $a l .{ }^{35}$ on the ${ }^{16} \mathrm{O}\left(\alpha, \alpha^{\prime}\right)$ reaction suggest that conside rable $E 2$ strength is distributed among several states located between $15-27 \mathrm{MeV}$ in ${ }^{16} \mathrm{O}$. Extensive investigations of $E 2$ strength distributions in light nuclei ${ }^{12,36-38}$ with radiative capture experiments indicate a fragmented distribution of $E 2$ strength extending over at least 10-15 MeV and encompassing a region below and above the GDR.

The broadening observed for the GQR in ${ }^{154} \mathrm{Sm}$ relative to ${ }^{144} \mathrm{Sm}$ is consistent with other experiments on deformed nuclei. Considerable broadening of the GQR was observed for deformed ${ }^{150} \mathrm{Nd}$ relative to spherical ${ }^{142} \mathrm{Nd}$ by electron scattering. ${ }^{39}$ From the published spectra we obtain ${ }^{26}$ a width for ${ }^{142} \mathrm{Nd}$ in agreement with our value and a broadening in Nd very similar to that for the Sm isotopes. A study of ${ }^{144-154} \mathrm{Sm}$ with inelastic proton scattering $^{29}$ revealed some differences in the giant resonance region, but the presence of the GDR and relatively poor statistics prevented any quantitative analysis. The authors interpret the ${ }^{144} \mathrm{Sm}$ 
data as containing two peaks in the giant resonance region separated by $\sim 2.5 \mathrm{MeV}$, consistent with our two-peak analysis discussed in Sec. VI. Our results would suggest that at least a portion of the higher energy peak seen in $\left(p, p^{\prime}\right)$ is part of the GQR. While the shapes of the spectra in the GR region are very similar, the $\left(p, p^{\prime}\right)$ data must be shifted $\sim 800 \mathrm{keV}$ to obtain agreement with the $\left(\alpha, \alpha^{\prime}\right)$ data. (The apparent agreement for GQR excitation energy in Table $\mathrm{V}$ is misleading, as only the low excitation portion of the asymmetric peak was used in the $\left(p, p^{\prime}\right)$ analysis. The upper portion of the peak was attributed to the GDR.)

No disagreement of this kind has been observed for other nuclei studied by both reactions. The $\left(\alpha, \alpha^{\prime}\right)$ data are the result of three independent runs where the GQR excitation energies were consistent to within $100 \mathrm{keV}$.

Narrow peaks below the GQR in excitation energy have been observed in several experiments. The present experiments indicate the presence of a group of states centered at about $13.3 \mathrm{MeV}$ and spread over a 1-2 MeV region in mass 56-60 nuclei. In ${ }^{58} \mathrm{Ni}$ a peak at $13.5 \mathrm{MeV}$ has been observed in $\left(p, p^{\prime}\right)^{34}$ where it was assigned $L=0$ or 2 and in $\left(l, d^{\prime}\right),{ }^{8}$ where no assignment was made. Proton scattering ${ }^{7}$ and inelastic electron scattering ${ }^{40}$ have led to an assignment of $L=3$ to a peak at about $13.4 \mathrm{MeV}$ in ${ }^{56} \mathrm{Fe}$, but $\left({ }^{3} \mathrm{He},{ }^{3} \mathrm{He}^{\prime}\right)$ results ${ }^{9}$ favor an $L=2$ assignment. We observe changes in the structure of this region with angle, which indicates the presence of more than one multipolarity, possibly explaining the discrepancy in assignments.

\section{CONCLUSIONS}

A giant resonance peak has been identified in 22 nuclei with mass numbers $36 \leqslant A \leqslant 208$. The peak lies at $E_{x}=60-65 / A^{1 / 3} \mathrm{MeV}$; for the lighter nuclei, there is a trend toward lower excitation energies. No GQR peak is apparent for nuclei with $A<32$. The angular distributions show a striking diffraction pattern and are fitted by DWBA calculations for $L=2$ transfer. Between $32 \%$ and $110 \%$ of the $E 2$ energy weighted sum rule is exhausted by the peak, and strong excitation by the isoscalar $\alpha$ particle would indicate that this is the isoscalar giant quadrupole resonance. These results are generally in agreement with other studies by inelastic electron, proton, deuteron, and ${ }^{3} \mathrm{He}$ scattering for position, width, and strength of the GQR. Such agreement adds weight to the identification of the state as the giant quadrupole resonance since the relative population of the different multipolarities is inherently quite different for the various reactions. The present results are also consistent with radiative capture measurements in $s-d$ shell and lighter nuclei where the quadrupole strength is observed to be spread over a wide energy range。

Now that many of the systematics of the GQR are established, the many differences and individual features must be investigated. The transition from a well-defined GQR in heavy nuclei to a diffuse and fragmented $E 2$ strength distribution in light nuclei is not fully understood. Structure in the region of the GQR is apparent for many nuclei; just below the GQR in the 40-60 mass region and possibly just above the GQR in the $A \approx 141-174$ mass region. In ${ }^{208} \mathrm{~Pb}$ considerable fine structure in the GQR region has been observed in several inelastic scattering studies and there are some indications, particularly for ${ }^{208} \mathrm{~Pb}$, that other multipoles may also be contributing to the peak. These individual nuclei now need to be studied in detail, certainly in several cases with good resolution, to identify the structure and to ascertain whether other multipoles may be important.

In conclusion, several things whose importance has become apparent during the course of these investigations should be pointed out. Analysis and interpretation of data on giant resonances obtained by inelastic scattering is hampered both by the large continuum underlying the peak and the possible presence of structure other than the giant resonance of interest. Careful experimental technique is required to insure the absence of spurious contributions in the spectra, and good statistics must be obtained to define the spectral shape. The use of $\alpha$ particles to excite the GQR has several advantages over other projectiles. The GDR is not excited in $\left(\alpha, \alpha^{\prime}\right)$, hence the $\alpha$ data may be used to ascertain the GDR contribution in proton and electron scattering where its contribution must be taken into account before the GQR properties can be obtained. The ratio of the peak height of the GQR to the nuclear continuum yield (for ${ }^{58} \mathrm{Ni}$ see Fig. 15) is more favorable in $\left(\alpha, \alpha^{\prime}\right)$ than in $\left(d, d^{\prime}\right)$, and the delineation between the peak and the background appears somewhat better. Recent experiments at this laboratory and elsewhere $^{35}$ suggest that a marked improvement in peak to background ratio can be obtained by utilizing still higher energy $\alpha$ beams. A careful comparison of several different reactions at this time provides the most reliable information on the composition of the observed giant resonances.

\section{ACKNOWLEDGMENTS}

We thank R. Weber for his assistance in taking and analyzing the data. Also, we appreciate invaluable discussions with Dr. T. Kishimoto about the general nature of the high-frequency oscillations. 
*Work supported in part by the National Science Foundation and the Robert A. Welch Foundation.

$\dagger$ Permanent address: Physics Department, Indiana University, Bloomington, Indiana.

${ }^{1}$ M. Goldhaber and E. Teller, Phys. Rev. 74, 1046 (1948).

${ }^{2}$ Proceedings of the International Conference on Photonuclear Reactions and Applications, Asilomar, 1973, edited by B. L. Berman (Lawrence Livermore Laboratory, Univ. of California, Livermore, 1973), M. Danos and E. G. Fuller, Annu. Rev. Nucl. Sci. 15, 29 (1965).

${ }^{3}$ A. Bohr and B. R. Mottelson, Neutron Capture GammaRay Spectroscopy (IAEA, Vienna, 1969); B. R. Mottelson, in Proceedings of the Fifteenth Solvay Conference in Physics, 1970 (Editions Stoops, Brussels, 1970).

${ }^{4} \mathrm{H}$. Uberall, Electron Scattering from Complex Nuclei (Academic, New York, 1971), Ch. 6.

${ }^{5}$ R. Pitthan and T. Walcher, Phys. Lett. 36B, 563 (1971).

${ }^{6}$ M. B. Lewis and F. E. Bertrand, Nucl. Phys. A196, 337 (1972).

${ }^{7}$ N. Marty, M. Morlet, A. Willis, V. Comparat, and R. Frascaria, Nucl. Phys. A238, 93 (1975).

${ }^{8}$ C. C. Chang, F. E. Bertrand, and D. C. Kocher, Phys. Rev. Lett. 34, 221 (1975).

${ }^{9}$ D. J. Horen, J. Arvieux, M. Buenerd, J. Cole, G. Perrin, and P. de Saintignon, Phys. Rev. C 11, 1247 (1975); J. Arvieux, M. Buenerd, J. Cole, P. de Saintignon, G. Perrin, and D. J. Horen, Nucl. Phys. A247, 238 (1975).

${ }^{10}$ L. L. Rutledge, Jr., and J. C. Hiebert, Phys. Rev. Lett. 32, 551 (1974).

${ }^{11}$ J. M. Moss, C. M. Rozsa, J. D. Bronson, and D. H. Youngblood, Phys. Lett. 53B, 51 (1974).

${ }^{12}$ E. Kuhlmann, E. Ventura, J. R. Calarco, D. G. Mavis, and S. S. Hanna, Phys. Rev. C 11, 1525 (1975); D. Branford, Particles and Nuclei $\underline{6}, 127$ (1973).

${ }^{13}$ L. Meyer-Schützmeister, W. Wharton, P. Debevec, R. E. Segel, and K. Raghunathan, Bull. Am. Phys. Soc. 20,565 (1975).

${ }^{14}$ A. M. Bernstein, Adv. Nucl. Phys. 3 , 325 (1969).

${ }^{15}$ J. M. Moss, C. M. Rozsa, D. H. Youngblood, J. D. Bronson, and A. D. Bacher, Phys. Rev. Lett. 34, 748 (1975). The Ca spectrum shown in this paper had sufficient ${ }^{16} \mathrm{C}$ contamination to distort the shape of the GQR.

${ }^{16}$ C. Pirart, M. Bosman, P. Leleux, P. C. Macq, and J. P. Meulders, Phys. Rev. C 10, 651 (1974); D. R. Brown, Ph.D. thesis, University of Washington, 1974 (unpublished).

${ }^{17}$ G. R. Satchler, Nucl. Phys. A195, 1 (1974); G. R. Satchler, Comm. Nucl. Part. Phys. 5, 145 (1972).

${ }^{18}$ Received from P. D. Kunz, University of Colorado.
${ }^{19}$ P. M. Endt and C. Van der Leun, Nucl. Phys. A214, 1 (1973).

${ }^{20}$ P. Stelson and L. Grodzins, Nucl. Data A1, 21 (1965).

${ }^{21}$ G. Bertsch and S. F. Tsai, Phys. Lett. $\overline{50 B}, 319$ (1974).

${ }^{22}$ E. C. Halbert, J. B. McGrory, G. R. Satchler, and J. Speth, Nucl. Phys. A245, 189 (1975).

${ }^{23}$ F. R. Buskirk, H. D. Graf, R. Pitthan, H. Theissen, O. Titoe, and T. Walcher, Phys. Lett. 42B, 194 (1972).

${ }^{24}$ M. Nagao and Y. Torizuka, Phys. Rev. Lett. $\underline{30}, 1068$ (1973).

${ }^{25}$ R. Pitthan, F. R. Buskirk, E. B. Dally, J. N. Dyer, and X. K. Maruyama, Phys. Rev. Lett. 33, 849 (1974).

${ }^{26}$ T. Kishimoto, J. M. Moss, D. H. Youngblood, J. D. Bronson, C. M. Rozsa, D. R. Brown, and A. D. Bacher, Phys. Rev. Lett. 35, 552 (1975).

${ }^{27}$ S. Fukuda and Y. Torizuka, Phys. Rev. Lett. 29, 1108 (1972).

${ }^{28}$ Y. Torizuka, K. Itoh, Y. M. Shin, Y. Kawazoe, H. Matsuzaki, and G. Takeda, Phys. Rev. C 11, 1174 (1975).

${ }^{29}$ D. J. Horen, F. E. Bertrand, and M. B. Lewis, Phys. Rev. C 9,1607 (1974).

${ }^{30} \mathrm{~F}$. Bertrand (private communication).

${ }^{31}$ R. Pitthan (private communication).

${ }^{32}$ A. Moalem, W. Benenson, and G. M. Crawley, Phys. Rev. Lett. 31, 482 (1973).

${ }^{33}$ D. C. Kocher, F. E. Bertrand, E. E. Gross, R. S. Lord, and E. Newman, Phys. Rev. Lett. 31, 1070 (1973); 32, 264(E) (1974); ORNL-5025, 1975 (unpublished), p. 61 .

${ }^{34} \mathrm{P}$. P. Singh and G. C. Yang, in Proceedings of the International Conference on Nuclear Structure and Spectroscopy, Amsterdam, 1974, edited by H. P. Blok and A. E. L. Dieperink (Scholar's Press, Amsterdam, 1974), Vol. I, p. 162.

${ }^{35}$ K. T. Knöpfle, G. J. Wagner, H. Breuer, M. Rosse, and C. Mayer-Böricke, Phys. Rev. Lett. 35,779 (1975).

${ }^{36} \mathrm{~J}$. R. Calarco, H. F. Glavish, E. Kuhlmann, S. S. Hanna, and P. M. Kurjan, Bull. Am. Phys. Soc. $\underline{19}$, 496 (1974).

${ }^{37}$ S. S. Hanna, H. F. Glavish, R. Avida, J. R. Calarco, E. Kuhlmann, and R. LaCama, Phys. Rev. Lett. 32 , 114 (1974).

${ }^{38}$ K. A. Snover, E. G. Adelberger, and D. R. Brown, Phys. Rev. Lett. 32, 1061 (1974).

${ }^{39}$ A. Schwierczinski, R. Frey, E. Sparner, H. Thiessen, and T. Walcher, Phys. Lett. 55B, 171 (1975).

${ }^{40}$ Y. Torizuka, Y. Kojima, T. Saito, K. Itoh, A. Nakada, S. Mitsunobu, M. Nagao, K. Hosoyama, S. Fukuda, and H. Miura, in Proceedings of the International Conference on Photonuclear Reactions and Applications, Asilomar, 1973 (see Ref. 2), p. 675. 